MATHEMATICS OF COMPUTATION

Volume 66, Number 220, October 1997, Pages 1407-1440

S 0025-5718(97)00878-8

\title{
ANALYSIS OF A FEM/BEM COUPLING METHOD FOR TRANSONIC FLOW COMPUTATIONS
}

\author{
H. BERGER, G. WARNECKE, AND W. L. WENDLAND
}

This work is dedicated to Professor Dr. Klaus Kirchgässner on the occasion of his 60th birthday

\begin{abstract}
A sensitive issue in numerical calculations for exterior flow problems, e.g. around airfoils, is the treatment of the far field boundary conditions on a computational domain which is bounded. In this paper we investigate this problem for two-dimensional transonic potential flows with subsonic far field flow around airfoil profiles. We take the artificial far field boundary in the subsonic flow region. In the far field we approximate the subsonic potential flow by the Prandtl-Glauert linearization. The latter leads via the Green representation theorem to a boundary integral equation on the far field boundary. This defines a nonlocal boundary condition for the interior ring domain. Our approach leads naturally to a coupled finite element/boundary element method for numerical calculations. It is compared with local boundary conditions. The error analysis for the method is given and we prove convergence provided the solution to the analytic transonic flow problem around the profile exists.
\end{abstract}

\section{Formulation of the problem}

1.1. The boundary value problem. Let $\Omega \subset \mathbb{R}^{2}$ be an open bounded domain surrounding a given simply connected wing section $P \subset \mathbb{R}^{2}$. The boundary of $\Omega$ consists of three parts

$$
\partial \Omega:=\Gamma_{\infty} \cup \Gamma_{P} \cup \Sigma,
$$

whose interiors are mutually disjoint and where $\Gamma_{\infty}$ and $\Gamma_{P}$ are disjoint closed Jordan curves connected by $\Sigma$. The curve $\Gamma_{\infty} \in \mathcal{C}^{\infty}$ is the artificial exterior boundary of $\Omega$ which is taken in order to obtain a bounded computational domain. The curve $\Gamma_{P}$ is the common boundary between $\Omega$ and the profile $P$, which has a corner, the trailing edge (TE), and is $\mathcal{C}^{\infty}$ otherwise. We denote by $\Sigma$ a slit in $\bar{\Omega}$, joining the trailing edge with $\Gamma_{\infty}$. The unbounded far field domain exterior to $\Gamma_{\infty}$ will be denoted by

$$
\Omega^{c}=\mathbb{R}^{2} \backslash \overline{\Omega \cup P} .
$$

The prolongation of the slit $\Sigma$ in $\Omega^{c}$ to infinity will be denoted by $\Sigma^{c}$. Without loss of generality, we assume that the travelling velocity is given by a constant vector field $\vec{v}_{\infty}$ which is parallel to the $x_{1}$-axis, see Figure 1.

Received by the editor August 13, 1993 and, in revised form, September 18, 1995.

1991 Mathematics Subject Classification. Primary 65N30, 68N38, 76H05, 49M10, 35L67.

Key words and phrases. Transonic full potential equation, artificial boundary conditions, finite elements, boundary elements, asymptotic error analysis.

The research reported in this paper was supported by the "Stiftung Volkswagenwerk". 


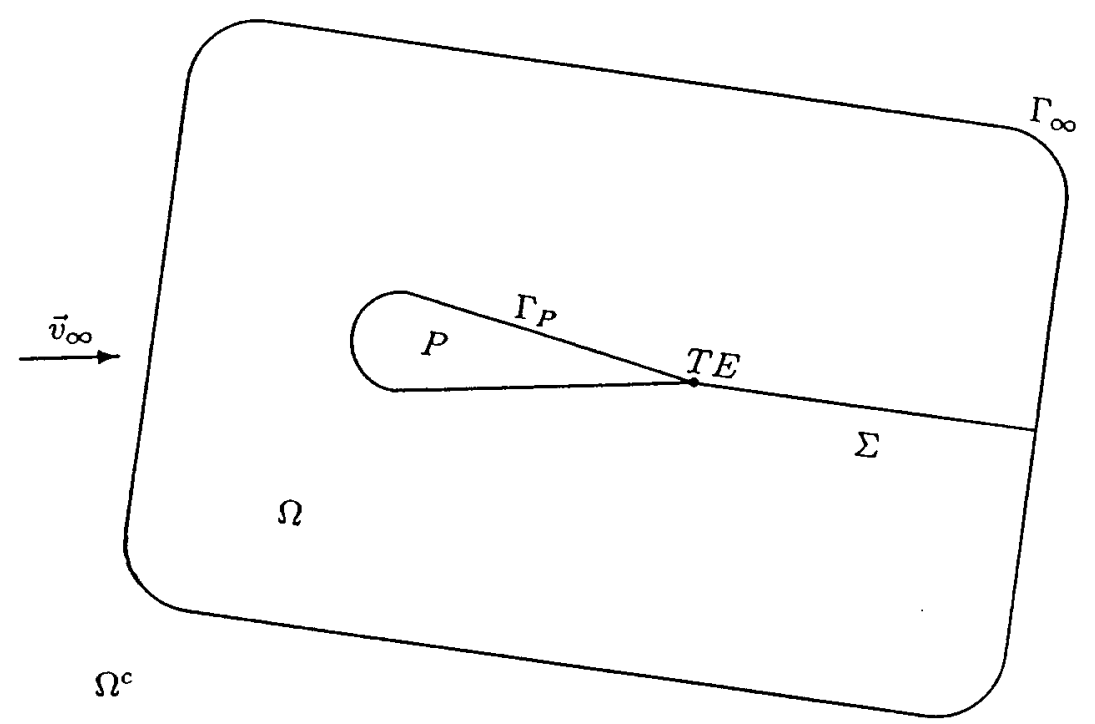

FIGURE 1. Computational domains for the coupling

In $\Omega$ we consider a stationary, compressible potential flow induced by a subsonic travelling velocity $\vec{v}_{\infty}$ at infinity. A simple model is the full potential equation, see Landau and Lifschitz [42],

$$
\operatorname{div}\left[\rho\left(|\nabla u|^{2}\right) \nabla u\right]=0 \quad \text { in } \Omega .
$$

This equation models inviscid, steady, isoenergetic, homentropic, planar flows of an ideal gas. Its generalized weak formulation admits transonic solutions with shock discontinuities in the velocity field. The equation (1.3) can be derived from conservation of mass, momentum and energy, see Berger et al. [9]. The density function $\rho(s)$ is obtained from Bernoulli's law and the assumption of homentropic flow. It is given as

$$
\rho\left(|\nabla u|^{2}\right)=\rho_{0}\left(1-\frac{\kappa-1}{2 a_{0}^{2}}|\nabla u|^{2}\right)^{\frac{1}{\kappa-1}} .
$$

Here $\kappa>1$ is the adiabatic gas constant, e.g. $\kappa=1.4$ for dry air. The constants $\rho_{0}, a_{0}$ are the density and the local speed of sound, respectively, for the motionless gas. The local speed of sound $a\left(|\nabla u|^{2}\right)$ is given by

$$
\left[a\left(|\nabla u|^{2}\right)\right]^{2}=a_{0}^{2} \rho_{0}^{1-\kappa}\left[\rho\left(|\nabla u|^{2}\right)\right]^{\kappa-1}=a_{0}^{2}-\frac{\kappa-1}{2}|\nabla u|^{2}
$$

and the local Mach number is $M:=\frac{|\nabla u|}{a}$. Note that the density $\rho(s)$ is only defined for $|\nabla u|^{2} \leq \frac{2 a_{0}^{2}}{k-1}$. This bound for the velocity will be assumed to hold in all further considerations.

The differential equation (1.3) with $\rho(s)$ given by (1.4) changes type at the sonic flow speed $|\nabla u|=a=a^{*}:=\left(\frac{2 a_{0}^{2}}{\kappa+1}\right)^{\frac{1}{2}}$, i.e. $M=1$. For $|\nabla u|<a^{*}$, i.e. $M<1$, the equation is elliptic and the velocity is subsonic; for $|\nabla u|>a^{*}$, i.e. $M>1$, it is hyperbolic and the velocity is supersonic. For sonic speed, $M=1$, the equation is degenerate, see Courant and Hilbert [23], Courant and Friedrichs [24]. 
In order to have a potential flow with circulation and lift we introduce the slit $\Sigma$ across which we assume that the velocity field $\nabla u$ is continuous, whereas the potential $u$ has a finite constant jump. This implies

$$
u^{+}-u^{-}=\beta \quad \text { and } \quad \partial_{n} u^{+}-\partial_{n} u^{-}=0 \quad \text { on } \Sigma .
$$

Here, $\vec{n}$ is a unit normal vector field on $\Sigma$ and $\partial_{n} u=\nabla u \cdot \vec{n}$. By $u^{+}, u^{-}$we denote the one-sided boundary values on $\Sigma$. The constant $\beta$ is an additional unknown and gives the circulation of the flow. To determine the jump $\beta$, we need the additional Kutta-Joukowski condition

$$
F(\beta):=\left|\nabla u^{+}\right|_{T E}^{2}-\left|\nabla u^{-}\right|_{T E}^{2}=0
$$

at the trailing edge $T E$ which follows from the requirement of continuous pressure there. Here we shall assume that the flow near $T E$ is subsonic which implies (1.6), too. (See Theorem 1.3.)

On the profile we impose the homogeneous Neumann boundary condition of non-penetration, which is equivalent to a vanishing mass flux, i. e.

$$
\partial_{n} u=0 \text { on } \Gamma_{P} .
$$

For completeness, we still need a boundary condition at $\Gamma_{\infty}$. It is generally assumed that a physically correct boundary condition for exterior flows is the requirement that the velocity $\vec{v}$ tends to the constant travelling velocity $\vec{v}_{\infty}$ at infinity. A simple and widely used method for approximating this condition is the following mass flux condition at $\Gamma_{\infty}$, i. e.

$$
\rho\left(|\nabla u|^{2}\right) \partial_{n} u=\rho\left(\left|\vec{v}_{\infty}\right|^{2}\right) \vec{v}_{\infty} \cdot \vec{n} \text { on } \Gamma_{\infty} .
$$

This is the condition used in Berger et al. [9]. A slight improvement of (1.8) can be obtained by replacing $\vec{v}_{\infty}$ on the right-hand side by $\vec{v}_{0_{\mid \Gamma_{\infty}}}$, where $\vec{v}_{0}$ is the velocity of the incompressible potential flow in $\Omega \cup \Omega^{c} \cup \Gamma_{\infty}$, see Berger et al. [8].

In this paper, however, we will consider the coupling with Prandtl-Glauert flow exterior to $\Gamma_{\infty}$. As is well known, the linear Prandtl-Glauert equation is

$$
\left(1-M_{\infty}^{2}\right) \varphi_{x_{1} x_{1}}+\varphi_{x_{2} x_{2}}=0 \quad \text { for } x=\left(x_{1}, x_{2}\right) \in \Omega^{c} .
$$

This is a linear approximation of (1.3). Here, the perturbation potential $\varphi$ is defined by

$$
\varphi:=u-\Psi \text { with } \Psi(x, \beta):=\vec{v}_{\infty} \cdot\left(x_{1}, x_{2}\right)+\frac{\beta}{2 \pi} \arctan \frac{\sqrt{1-M_{\infty}^{2}} \cdot x_{2}}{x_{1}},
$$

where $M_{\infty}$ is the Mach number at infinity, see Zierep [57] for details. For the arctan the branch must be chosen in such a way that the jump occurs at the slits $\Sigma$ and $\Sigma^{c}$ which meet at $\Gamma_{\infty}$. Of course, we assume that the perturbation velocity field $\nabla \varphi$ is continuous across the slit $\Sigma^{c}$ and the pertubation potential $\varphi$, by definition (1.10), is continuous across $\Sigma^{c}$, too. This gives

$$
\varphi^{+}-\varphi^{-}=0 \quad \text { and } \quad \partial_{n} \varphi^{+}-\partial_{n} \varphi^{-}=0 \quad \text { on } \Sigma^{c} .
$$

For the perturbation velocity $\nabla \varphi$ we prescribe the radiation condition at infinity,

$$
\nabla \varphi(x) \rightarrow 0 \quad \text { for }|x| \rightarrow \infty .
$$

In addition, we need two more transmission conditions for the coupling of $u$ with $\varphi$ at $\Gamma_{\infty}$. Denote by $\vec{n}=\left(n_{1}, n_{2}\right)$ the outer unit normal field on $\Gamma_{\infty}$, and set $\partial_{n} u=\nabla u \cdot \vec{n}$. The first transmission condition is simply obtained by definition 
(1.10) and continuity across $\Gamma_{\infty}$. For the second transmission condition we require equality of the mass flux $\rho\left(|\nabla u|^{2}\right) \partial_{n} u$ to the corresponding expression defined by linearization about the Prandtl-Glauert solution. The latter leads for the density to $\rho \approx \rho_{\infty}:=\rho\left(\left|\vec{v}_{\infty}\right|^{2}\right)$ in $\Omega^{c}$. The coupling conditions become

$$
u=\varphi+\Psi \quad \text { on } \Gamma_{\infty}
$$

and

$$
\begin{aligned}
& \rho\left(|\nabla u|^{2}\right) \nabla u \cdot \vec{n} \\
& \quad=\rho_{\infty}\left\{\nabla \Psi \cdot \vec{n}+\left(1-M_{\infty}^{2}\right) \varphi_{x_{1}} n_{1}+\varphi_{x_{2}} n_{2}\right\} \quad \text { on } \Gamma_{\infty} .
\end{aligned}
$$

Collecting the equations (1.3), (1.5), (1.6), (1.7), (1.9), (1.11), (1.12) and the coupling conditions (1.13), (1.14), we get the following system of equations, boundary and transmission conditions:

\section{Coupled boundary value problem}

Find the functions $u, \varphi$ in appropriate function spaces and the constant $\beta \in \mathbb{R}$ satisfying the

Interior full potential problem,

$$
\begin{aligned}
\operatorname{div}\left[\rho\left(|\nabla u|^{2}\right) \nabla u\right] & =0 & & \text { in } \Omega, \\
\partial_{n} u & =0 & & \text { on } \Gamma_{p}, \\
u^{+}-u^{-} & =\beta & & \text { on } \Sigma, \\
\partial_{n} u^{+}-\partial_{n} u^{-} & =0 & & \text { on } \Sigma, \\
F(\beta)=\left|\nabla u^{+}\right|_{T E}^{2}-\left|\nabla u^{-}\right|_{T E}^{2} & =0 ; & &
\end{aligned}
$$

Exterior Prandtl-Glauert problem,

$$
\begin{aligned}
\left(1-M_{\infty}^{2}\right) \varphi_{x_{1} x_{1}}+\varphi_{x_{2} x_{2}} & =0 & & \text { in } \Omega^{c} \\
\nabla \varphi & =o(1) & & \text { for }|x| \rightarrow \infty \\
\varphi^{+}-\varphi^{-} & =0 & & \text { on } \Sigma^{c} \\
\partial_{n} \varphi^{+}-\partial_{n} \varphi^{-} & =0 & & \text { on } \Sigma^{c}
\end{aligned}
$$

$\underline{\text { Coupling conditions, }}$

$$
u=\varphi+\Psi \quad \text { on } \Gamma_{\infty}
$$

and

$$
\begin{aligned}
& \rho\left(|\nabla u|^{2}\right) \partial_{n} u \\
& \quad=\rho\left(\left|\vec{v}_{\infty}\right|^{2}\right)\left\{\nabla \Psi \cdot \vec{n}+\left(\left(1-M_{\infty}^{2}\right) \varphi_{x_{1}}, \varphi_{x_{2}}\right) \cdot \vec{n}\right\} \quad \text { on } \Gamma_{\infty} .
\end{aligned}
$$

Note that, for a solution $(u, \varphi, \beta)$ of this coupled problem (1.15)-(1.18), one also has the solution $(u+c, \varphi+c, \beta)$ with an arbitrary constant $c$. We therefore fix this constant by the requirement

$$
\int_{\Gamma_{\infty}} \varphi d s=0 .
$$

With the Euler equations in $\Omega$ instead of the full potential model (1.15), Sofronov and Tscincov present a similar coupling formulation in [55]. 
1.2. The weak formulation. Let $W^{s, p}(\Omega), \quad s \in \mathbb{R}, \quad p \in[1, \infty]$ and $W^{s, p}\left(\Gamma_{\infty}\right)$ be the usual Sobolev spaces equipped with norms $\|\cdot\|_{W^{s, p}(\Omega)}$ and $\|\cdot\|_{W^{s, p}\left(\Gamma_{\infty}\right)}$, respectively. We define $\langle\cdot, \cdot\rangle$ to be the duality pairing between $H^{s}\left(\Gamma_{\infty}\right):=W^{s, 2}\left(\Gamma_{\infty}\right)$ and the dual space $H^{-s}\left(\Gamma_{\infty}\right)$ with respect to the $L^{2}\left(\Gamma_{\infty}\right)$ inner product,

$$
\langle\chi, \psi\rangle:=\int_{\Gamma_{\infty}} \chi(s) \psi(s) d s \text { for all }(\chi, \psi) \in H^{s}\left(\Gamma_{\infty}\right) \times H^{-s}\left(\Gamma_{\infty}\right) .
$$

We further introduce the spaces

$$
\begin{aligned}
V & :=\left\{v \in W^{1,2}(\Omega) \mid v^{+}-v^{-}=\beta \text { on } \Sigma, \beta \in \mathbb{R}\right\}, \\
V^{0} & :=\left\{v \in W^{1,2}(\Omega) \mid v^{+}-v^{-}=0 \text { on } \Sigma\right\} \\
H & :=\left\{\psi \in H^{-\frac{1}{2}}\left(\Gamma_{\infty}\right) \mid \int_{\Gamma_{\infty}} \psi d s=0\right\}
\end{aligned}
$$

and the set of admissible functions

$$
K_{s_{0}}:=\left\{\left.v \in V|| \nabla v\right|^{2} \leq s_{0}<\frac{2 a_{0}^{2}}{\kappa-1} \quad \text { a.e. in } \Omega\right\} .
$$

$K_{s_{0}}$ is a closed, convex subset of $V$ in $W^{1,2}(\Omega)$. In order to simplify the notations we define the following nonlinear form

$$
a(u \mid v, w):=\int_{\Omega} \rho\left(|\nabla u|^{2}\right) \nabla v \cdot \nabla w d x \quad \text { for all triplets }(u, v, w) \in K_{s_{0}} \times V \times V .
$$

For solving the exterior problem (1.16) we shall use a boundary potential formulation based on the Green representation theorem. To this end, one transforms the Prandtl-Glauert equation in (1.16) with constant coefficients into Laplace's equation and then uses classical potential theory. Any sufficiently smooth solution $\varphi$ satisfying (1.16) has the behavior

$$
\varphi(x)=\frac{\widetilde{\beta}}{2} \log \left(\frac{x_{1}^{2}}{1-M_{\infty}^{2}}+x_{2}^{2}\right)+\varphi_{\infty}+o(1) \quad \text { for }|x| \rightarrow \infty,
$$

with constant $\varphi_{\infty}$. One obtains with the fundamental solution

$$
G(x, y):=\frac{1}{2 \pi} \log \left|\left(\frac{1}{\sqrt{1-M_{\infty}^{2}}}\left[x_{1}-y_{1}\right],\left[x_{2}-y_{2}\right]\right)\right|
$$

and the kernel of the double layer potential

$$
K(x, y):=\frac{1}{2 \pi} \frac{\left(\left[x_{1}-y_{1}\right],\left[x_{2}-y_{2}\right]\right) \cdot \vec{n}(y)}{\left|\left(\frac{1}{\sqrt{1-M_{\infty}^{2}}}\left[x_{1}-y_{1}\right],\left[x_{2}-y_{2}\right]\right)\right|^{2}}
$$


the Green representation formula in the form

$$
\begin{aligned}
\varphi(x)= & \frac{\varepsilon}{\sqrt{1-M_{\infty}^{2}}} \int_{\Gamma_{\infty}}\left\{\left(1-M_{\infty}^{2}\right) \varphi_{y_{1}} n_{1}(y)+\varphi_{y_{2}} n_{2}(y)\right\} G(x, y) d s_{y} \\
& +\frac{\varepsilon}{\sqrt{1-M_{\infty}^{2}}} \int_{\Gamma_{\infty}} \varphi(y) K(x, y) d s_{y}+\varepsilon \varphi_{\infty}
\end{aligned}
$$

with $\varepsilon=1$ for $x=\left(x_{1}, x_{2}\right) \in \Omega^{c}$ and $\varepsilon=2$ for $x \in \Gamma_{\infty}$. In the latter case, (1.25) defines a boundary integral equation relating the Cauchy data $\left(1-M_{\infty}^{2}\right) \varphi_{x_{1}} n_{1}(x)+$ $\varphi_{x_{2}} n_{2}(x)$ and $\varphi(x)$ to each other. It can easily be shown that (1.25) corresponds to the choice of zero for the additional constant mentioned at the end of Section 1.1 and used in the definition of $H$ in (1.20). We introduce the co-normal derivative of $\varphi$ on $\Gamma_{\infty}$ by

$$
\lambda(x):=\rho\left(\left|\vec{v}_{\infty}\right|^{2}\right)\left\{\left(1-M_{\infty}^{2}\right) \varphi_{x_{1}} n_{1}(x)+\varphi_{x_{2}} n_{2}(x)\right\}
$$

and define the boundary integral operators of single and double layer potentials

$$
\begin{aligned}
\mathcal{V} \lambda(x) & :=-\int_{\Gamma_{\infty}} \frac{2 G(x, y)}{\rho\left(\left|\vec{v}_{\infty}\right|^{2}\right) \sqrt{1-M_{\infty}^{2}}} \lambda(y) d s_{y}, \\
\mathcal{K} \varphi(x) & :=\int_{\Gamma_{\infty}} \frac{2 K(x, y)}{\sqrt{1-M_{\infty}^{2}}} \varphi(y) d s_{y} .
\end{aligned}
$$

Thus, for $x \in \Gamma_{\infty}$, we may write (1.25) in short as

$$
\varphi(x)+\mathcal{V} \lambda(x)-\mathcal{K} \varphi(x)=2 \varphi_{\infty} .
$$

For smooth $\Gamma_{\infty}$ we have the following well known result, see Hsiao and Wendland [38].

Lemma 1.1. For a $\mathcal{C}^{\infty}$-boundary $\Gamma_{\infty}$, and any $\sigma \in \mathbb{R}$, the boundary integral operators

$$
\begin{array}{lll}
\mathcal{V}: & H^{\sigma-\frac{1}{2}}\left(\Gamma_{\infty}\right) \rightarrow H^{\sigma+\frac{1}{2}}\left(\Gamma_{\infty}\right) \quad \text { and } \\
\mathcal{K}: H^{\sigma+\frac{1}{2}}\left(\Gamma_{\infty}\right) \rightarrow H^{\sigma+\frac{3}{2}}\left(\Gamma_{\infty}\right)
\end{array}
$$

are continuous.

Further, we define the bilinear forms

$$
b(\lambda, \psi):=\langle\mathcal{V} \lambda, \psi\rangle \quad \text { and } \quad d(\lambda, \psi):=\langle\mathcal{K} \lambda, \psi\rangle
$$

for $\lambda, \psi \in H^{-\frac{1}{2}}\left(\Gamma_{\infty}\right)$ and, finally with the function $\Psi=\Psi(x, \beta)$ given by (1.10), for any $\beta \in \mathbb{R}$ the functionals

$$
\ell_{1}(v, \beta):=\rho_{\infty}\langle\nabla \Psi(\cdot, \beta) \cdot \vec{n}, v\rangle
$$

and

$$
\ell_{2}(\psi, \beta):=\langle(\mathcal{I}-\mathcal{K}) \Psi(\cdot, \beta), \psi\rangle
$$

for all $v \in W^{\sigma, p}(\Omega)$ and all $\psi \in H^{-\frac{1}{2}+\sigma}\left(\Gamma_{\infty}\right)$. For every fixed $\beta \in \mathbb{R}$, these are linear and bounded functionals. 
Lemma 1.2. The nonlinear form $a(\cdot \mid \cdot, \cdot)$ satisfies the following estimates:

$$
\begin{aligned}
|a(u \mid v, w)| & \leq \rho_{0}\|v\|_{W^{1,2}(\Omega)}\|w\|_{W^{1,2}(\Omega)} \\
& \text { for all }(u, v, w) \in K_{s_{0}} \times V \times V, \\
|a(u \mid u, w)-a(v \mid v, w)| & \leq C\|u-v\|_{W^{1,2}(\Omega)}\|w\|_{W^{1,2}(\Omega)} \\
& \text { for all }(u, v, w) \in K_{s_{0}} \times K_{s_{0}} \times V .
\end{aligned}
$$

Moreover, if we choose $s_{0}<\frac{2 a_{0}^{2}}{\kappa+1}$, then we obtain uniform monotonicity for these purely subsonic flow fields, i.e.

$$
\begin{aligned}
a(u \mid u, u-v)-a(v \mid v, u-v) \geq & \gamma_{1}\|u-v\|_{W^{1,2}(\Omega)}^{2} \\
& \text { for all }(u, v) \in K_{s_{0}} \times K_{s_{0}} .
\end{aligned}
$$

For a $\mathcal{C}^{\infty}$-boundary $\Gamma_{\infty}$ and $\sigma \in \mathbb{R}$ there exists a constant $C$ such that the form $b(\lambda, \psi)$ satisfies

$$
|b(\lambda, \psi)| \leq C\|\lambda\|_{H^{\sigma-\frac{1}{2}}\left(\Gamma_{\infty}\right)}\|\psi\|_{H^{-\sigma-\frac{1}{2}}\left(\Gamma_{\infty}\right)} .
$$

Moreover, there exists a constant $\gamma_{2}>0$ such that

$$
b(\psi, \psi) \geq \gamma_{2}\|\psi\|_{H^{-\frac{1}{2}}\left(\Gamma_{\infty}\right)}^{2} \quad \text { for all } \psi \in H .
$$

For the linear forms we have with some constant $C$ the estimates

$$
\begin{aligned}
\left|\ell_{1}(v, \beta)\right| & \leq\left|\varrho_{\infty}\right|\left\{\left|\vec{v}_{\infty}\right|+C|\beta|\right\}\|v\|_{H^{-\frac{1}{2}}\left(\Gamma_{\infty}\right)}, \\
\left|\ell_{1}\left(v, \beta_{1}\right)-\ell_{1}\left(v, \beta_{2}\right)\right| & \leq C\left|\beta_{1}-\beta_{2}\right|\|v\|_{H^{-1}\left(\Gamma_{\infty}\right)},
\end{aligned}
$$

and

$$
\begin{aligned}
\left|\ell_{2}(\psi, \beta)\right| & \leq C\left\{\left|\vec{v}_{\infty}\right|+|\beta|\right\}\|\psi\|_{H^{-\frac{1}{2}}\left(\Gamma_{\infty}\right)}, \\
\left|\ell_{2}\left(\psi, \beta_{1}\right)-\ell_{2}\left(\psi, \beta_{2}\right)\right| & \leq C\left|\beta_{1}-\beta_{2}\right|\|\psi\|_{H^{-\frac{1}{2}}\left(\Gamma_{\infty}\right)} .
\end{aligned}
$$

Proof. The inequalities (1.31) follow from the facts that $|\rho(s)| \leq \rho_{0}$ and $\mid \rho(s)+$ $2 s \rho^{\prime}(s) \mid \leq c$ hold for all $s \in\left[0, s_{0}\right]$. The coerciveness inequality (1.32) is a consequence of $\rho(s)+2 s \rho^{\prime}(s) \geq \gamma_{1}>0$ for all $s_{0} \in\left[0, \frac{2 a_{0}^{2}}{\kappa+1}\right)$. The properties of the form $b$ are shown by Hsiao and Wendland in [38], the continuity estimates for $\ell_{1}$ follow from (1.10) and those for $\ell_{2}$ from Lemma 1.1.

Remark. The coerciveness inequality (1.34) and the mapping properties of $\mathcal{V}$ and $\mathcal{K}: H^{\sigma+\frac{1}{2}}\left(\Gamma_{\infty}\right) \rightarrow H^{1}\left(\Gamma_{\infty}\right)$ in (1.29) remain valid with $|\sigma| \leq \frac{1}{2}$ even for Lipschitz curves $\Gamma_{\infty}$ due to Costabel [22].

If we multiply equation (1.28) by a test function $\psi \in H$ and integrate over $\Gamma_{\infty}$, we obtain

$$
\langle\mathcal{V} \lambda, \psi\rangle+\langle\varphi, \psi\rangle-\langle\mathcal{K} \varphi, \psi\rangle=b(\lambda, \psi)+\langle\varphi, \psi\rangle-d(\varphi, \psi)=0 \quad \text { for all } \psi \in H .
$$

This is the weak formulation for the boundary integral equation (1.28).

The weak formulation for a solution of problem (1.15) can be obtained by the usual variational approach.

Find $u \in K_{s_{0}}$ and $\beta \in \mathbb{R}$ such that

$$
a(u \mid u, v)-\left\langle\rho\left(|\nabla u|^{2}\right) \partial_{n} u, v\right\rangle=0 \quad \text { for all } v \in V^{0} .
$$


The coupling of the weak formulation (1.36) in $\Omega$ and (1.35) in $\Omega^{c}$ can be obtained with $(1.17),(1.18)$ in variational form via the flux balance (1.14) through $\Gamma_{\infty}$ and reads:

\section{Variational problem}

Find the three quantities $(u, \lambda, \beta) \in K_{s_{0}} \times H \times \mathbb{R}$ such that

$$
\left\{\begin{array}{l}
a(u \mid u, v)-\langle\lambda, v\rangle=\ell_{1}(v, \beta) \quad \text { for all } v \in V^{0} \\
b(\lambda, \psi)+\langle u, \psi\rangle-d(u, \psi)=\ell_{2}(\psi, \beta) \text { for all } \psi \in H^{-\frac{1}{2}}\left(\Gamma_{\infty}\right) \text { and } \\
F(\beta)=\left|\nabla u^{+}\right|_{T E}^{2}-\left|\nabla u^{-}\right|_{T E}^{2}=0 .
\end{array}\right.
$$

Note that the solvability of this problem can be proved rigorously, up to now, only in the subsonic case. For transonic flows, however, we shall assume the existence of an appropriate solution. This includes that an appropriate choice of $s_{0}$ is possible.

In the following section we will discuss the Kutta-Joukowski condition in (1.37).

1.3. The Kutta-Joukowski condition. For subsonic flows, i.e. with sufficiently small $\vec{v}_{\infty},(1.36)$ is a nonlinear elliptic Neumann problem in variational form. The set of admissible functions (1.21) excludes an arbitrary growth of $|\nabla u|$ at the trailing edge $T E$. The solution of a linear and also a nonlinear elliptic problem would in general develop a singular growth of $|\nabla u|$ at the reentrant corner $T E$ if $\beta$ is not chosen appropriately. In case of a singularity, however, there would be a supersonic region at $T E$ which we exclude for physical reasons.

Theorem 1.3. Let the trailing edge angle $\omega$ satisfy $0<\omega<\pi$. Let $(u, \lambda, \beta) \in$ $K_{s_{0}} \times H \times \mathbb{R}$ be the variational solution satisfying

$$
\begin{aligned}
a(u \mid u, v)-\langle\lambda, v\rangle & =\ell_{1}(v, \beta) & & \text { for all } v \in V^{0}, \\
b(\lambda, \psi)+\langle u, \psi\rangle-d(u, \psi) & =\ell_{2}(\psi, \beta) & & \text { for all } \psi \in H .
\end{aligned}
$$

Let us further assume that there exists some bound $s_{T E} \in\left(0, \frac{2 a_{0}^{2}}{\kappa+1}\right)$ and a radius $r_{0}>0$ such that around the trailing edge

$$
|\nabla u(x)|^{2} \leq s_{T E} \quad \text { for all } x \in \mathcal{U}:=\left\{x \in \bar{\Omega}|| x-T E \mid<r_{0}\right\},
$$

i.e. the flow is subsonic around TE.

Then the velocity field $\nabla u$ is Hölder continuous in $\mathcal{U}$ and already satisfies the Kutta-Joukowski condition

$$
\nabla u_{\mid T E}^{+}=\nabla u_{\left.\right|_{T E}}^{-} .
$$

The proof of this theorem will be given in the paper [21].

At a first glance, for a variational solution $u$ in $W^{1,2}(\Omega)$, the point condition (1.39) seems not to be well defined. Since, on the other hand, it also means that the stress intensity factor associated with $T E$ and the subsonic flow is to be zero, this is a continuous functional on the solution space. For subsonic Prandtl-Glauert linearizations, this requirement determines the circulation $\beta$ uniquely. In the paper [21] this relation will be used. Incorporating (1.39) into the solution space yields a fast deterioration of the condition numbers of the associated discretizations. Here, however, we analyze the method described in [10] where a simple relaxation procedure is used enforcing (1.39) in every iteration step. 
For purely subsonic cascade flow, Feistauer et al. in [29] also use the KuttaJoukowski condition for finding the appropriate finite element solution providing circulation.

\section{EXISTENCE, UNIQUENESS AND THE ENTROPY CONDITION}

The coupled boundary value problem (1.15)-(1.18) is an approximation to the:

\section{Exterior Boundary Value Problem}

Find an appropriate function $u$ with $|\nabla u|^{2}<\frac{2 a_{0}^{2}}{\kappa-1}$ satisfying

$$
\begin{aligned}
\operatorname{div}\left[\rho\left(|\nabla u|^{2}\right) \nabla u\right] & =0 & & \text { in } \Omega \cup \Gamma_{\infty} \cup \Omega^{c}, \\
\partial_{n} u & =0 & & \text { on } \Gamma_{p}, \\
u^{+}-u^{-} & =\beta & & \text { on } \Sigma \cup \Sigma^{c}, \\
\partial_{n} u^{+}-\partial_{n} u^{-} & =0 & & \text { on } \Sigma \cup \Sigma^{c}, \\
\vec{v}(x) \longrightarrow \vec{v}_{\infty} & & & \text { for }|x| \rightarrow \infty, \\
F(\beta)=\left|\nabla u^{+}\right|_{T E}^{2}-\left|\nabla u^{-}\right|_{T E}^{2} & =0 . & &
\end{aligned}
$$

For the subsonic solution to this exterior problem see Bers [11] and Bojarski [13]. Here, however, we allow the solution to be transonic. Moreover, the difference to the coupled problem (1.15)-(1.18) lies in the fact that there we take the linearization on $\Omega^{c}$ and the corresponding coupling boundary conditions on $\Gamma_{\infty}$. Under the restriction

$$
\|\nabla u\|_{L^{\infty}(G)}<a^{*} \quad \text { in the subdomain } G \subset \Omega \cap \Gamma_{\infty} \cup \Omega^{c},
$$

the problem (2.1) is elliptic there, i.e. the flow is subsonic on $G$. A unique solution exists for all $\vec{v}_{\infty}$ small enough to imply that (2.2) holds everywhere. This result has a long history, see e.g. Frankl and Keldysh [34], Bers [11] and the references given there, Bojarski [13], Brezis and Stampacchia [14], Ciavaldini, Pogu and Tournemine [20], [19], [51] and Feistauer and Nečas [31]; most authors treated the problem in the stream function formulation. The upper bound (2.2) as a global condition depends on $\vec{v}_{\infty}$ and the geometry (thickness and form) of the profile $P$. Therefore, it has not been possible up to now to explicitly give a priori conditions that imply (2.2).

Due to the jump across the slit $\Sigma \cup \Sigma^{c}$, the solution is not in $H^{1}$ in a neighbourhood of the slit. It is locally only in $L^{2}$ because $\nabla u$ is a locally bounded measure that has a singular part (see Federer [28]) supported on $\Sigma \cup \Sigma^{c}$ which is weighted by the jump strength $\beta$. But, the fact that the coupling conditions imply $\nabla u^{+}=\nabla u^{-}$ on $\Sigma \cup \Sigma^{c}$ means that the absolutely continuous part of the measure $\nabla u$ lies in $L^{2}$ even across $\Sigma \cup \Sigma^{c}$. The singular part is unavoidable in a potential formulation with circulation in domains that are not simply connected, however, only the absolutely continuous part of $\nabla u$ defines the velocity field.

Elliptic regularity theory, which is applicable wherever (2.2) holds, gives interior $H^{2}$ regularity of the solution there. Across the coupling boundary $\Gamma_{\infty}$, even if $\Gamma_{\infty}$ is smooth, one cannot expect more than $H^{1}$, as is well known for elliptic problems with discontinuous coefficients, see Fix and Strang [33] for a simple example.

The question of regularity at the trailing edge is complicated by the fact that we put the slit $\Sigma$ there. This was done entirely for numerical convenience. For the moment we may suppose that $\Sigma$ touches the profile somewhere else. Then generically 
the solution will have a corner singularity at $T E$ and be only in $H^{1}$ there. Based on results for the linear theory by Djaoua [27], the stream function formulation by Ciavaldini, Pogu and Tournemine [20], [19], and 3-D potential flows by Dauge and Pogu [26] it is shown in [21] that the Kutta-Joukowski condition selects the unique value $\beta$ such that the solution has $H^{2}$ regularity up to the boundary also at $T E$.

Now let us take a brief look at the existence of subsonic solutions of the weak, coupled boundary value problem (1.37). For the subsonic case after subtracting the circulation term, the problem (1.37) can be seen as an exterior nonlinear elliptic problem having discontinuous coefficients across $\Gamma_{\infty}$. Via Kelvin transformation, this problem becomes an interior corresponding nonlinear elliptic problem for which in the subsonic case existence follows as in [15], yielding existence for (1.37). To treat the full problem with unknown circulation $\beta$, also in the transonic case, we present a nonlinear iteration in function spaces satisfying the Kutta-Joukowski condition (1.39) by correcting the iterates in every step with appropriate $\beta$-values to enforce regularity at $T E$ as described in [10]. For subsonic flows, a similar coupling involving the stream function formulation with Dirichlet conditions was treated in [29].

2.1. The entropy condition. For the case in which the geometry of the problem and the boundary conditions lead to a locally supersonic flow near the profile, the situation changes quite dramatically. In the supersonic regions the equation (1.3) becomes hyperbolic and the monotonicity property (1.32) is lost. In this case the existence of solutions to (2.1) is still open, even for problems with bounded domains, see Feistauer and Nečas [31] and Morawetz [48].

A further complication comes from the fact that mathematical analysis by Morawetz [45], [46], [47], as well as physical and numerical experience with the problem, show that one generally has to expect solutions that have discontinuous derivatives $\nabla u$, i.e. contain shocks. Hence, one has to consider weak solutions which in turn lead to non-uniqueness and the existence of non-physical solutions to the variational problem (2.1), see Nečas [50]. This fact, which is well known from the related theory of systems of conservation laws, see Smoller [54], is the reason why Bristeau et al. in [15], [16], [17] need to supplement the problem with an additional admissibility condition for generalized solutions. We will concentrate here on the specific numerical entropy condition used for our numerical implementation which we adopted from Glowinski and Pironneau [36]. Further discussion on admissibility of transonic shocks may be found in Nečas [50], Keyfitz and Warnecke [40], and Warnecke [56]. Due to the assumption of isentropy, the shocks in transonic potential flow conserve entropy but not momentum.

The practical entropy condition we have used requires that the divergence of the flow field must be bounded from above, i.e.

$$
\operatorname{div} \nabla u \leq B \text { with an appropriate constant } B \in \mathbb{R} .
$$

In Göhner and Warnecke [37] it is shown that this inequality is equivalent to the solution with compressive shocks and is violated by the non-physical expansion shocks. In weak form this means that the inequality

$$
-\int_{\Omega} \nabla u \cdot \nabla \psi d x \leq B \int_{\Omega} \psi d x \text { for all } \psi \in C_{0}^{\infty}(\Omega) \text { with } \psi \geq 0
$$

must hold. The implementation of (2.4) via penalization is shown in Section 4 . 


\section{The Coupled FEM-BEM Formulation of the Problem}

In order to discretize the coupled problem (1.37), we approximate the domain $\Omega$ by a family of polygonal domains $\Omega_{h}$ where $h$ denotes the parameter of meshwidth. The outer boundary $\Gamma_{\infty}^{h}$ of $\Omega_{h}$ is supposed to be a polygonal curve with nodes on $\Gamma_{\infty}$ which approximates $\Gamma_{\infty}$. In the same way we define $\Gamma_{P}^{h}$ as the approximation of the profile boundary $\Gamma_{P}$. Without loss of generality we may assume that the slit $\Sigma$ is already a part of the boundary of $\Omega_{h}$. Together with $\Omega_{h}$ we introduce now a family of regular triangulations $\left\{\mathcal{T}_{h}\right\}_{h>0}$ with $\mathcal{T}_{h}:=\bigcup_{i \in \mathbb{D}} T_{i}$ where $\mathbb{D}$ is a finite subset of the natural numbers $\mathbb{N}$. The nodal points of the triangulation are denoted by $p_{i}, i=1, \ldots, N$. For convenience we assume that the nodes on the slit $\Sigma$ will be taken to be the first $2 L$ points, i.e. $p_{j}^{+}, p_{j}^{-}, j=1, \ldots L$, with $p_{j}^{+}$and $p_{j}^{-}$having the same coordinates but characterizing the limits from above and below, respectively. By $w_{h_{i}}, i=1, \ldots, N$, we denote the usual piecewise linear hat functions satisfying $w_{h_{i}}\left(p_{j}\right)=\delta_{i j}, i, j=1, \ldots, N$, which form a basis of nonnegative functions for the piecewise linear continuous finite elements. By $A_{i}$ we denote the areas of the corresponding supports, i.e. $A_{i}=$ meas $\left(\operatorname{supp} w_{h_{i}}\right), i=1, \ldots, N$.

For a triangle $T_{i} \in \mathcal{T}_{h}$ let $\alpha_{i}$ denote the smallest angle. We say that a family of regular triangulations $\left\{\mathcal{T}_{h}\right\}_{h>0}$ satisfies the angle property if there is a minimal angle $\alpha>0$ such that for any $h>0$ and any $T_{i} \in \mathcal{T}_{h}$ one has $\alpha_{i} \geq \alpha$.

We denote by $S_{i}$ the segments on $\Gamma_{\infty}^{h}$ given by the edges of the boundary triangles $T_{i}$ of $\mathcal{T}_{h}$. By $\left\{\mathcal{S}_{h}\right\}_{h>0}$ with $\mathcal{S}_{h}:=\bigcup_{i \in \mathbb{B}}^{\infty} S_{i}$ we denote the corresponding induced family of polygonal approximations of the boundary $\Gamma_{\infty}^{h}$, where $\mathbb{B}$ is a finite subset of $\mathbb{N}$. Without loss of generality we assume that our triangulation $\Omega_{h}$ is chosen such that the corresponding family $\left\{\mathcal{S}_{h}\right\}_{h>0}$ guarantees the validity of an inverse assumption, see Ciarlet [18, (3.2.28)]. This implies inverse estimates, see Ciarlet [18, Theorem 3.2.6]. The error analysis is carried out for a Galerkin discretization, also for the boundary element method. However, we implemented the boundary element method using point collocation. Then it can be shown that the asymptotic error estimates used in Section 5 still remain valid due to [2]. The family $\left\{\mathcal{T}_{h}\right\}_{h>0}$ itself does not need to be quasiuniform. For all further considerations, the parameter $h$ will stand for the maximum diameter of all triangles $T_{i} \in \mathcal{T}_{h}$. Let $\mathcal{C}^{0}\left(\bar{\Omega}_{h}\right)$ denote the set of all continuous functions on $\Omega_{h}$, having one-sided limits on the slit $\Sigma$ and $\partial \Omega_{h}$, respectively. For the discretization of (1.37) we introduce the following finite-dimensional spaces on the polygonal domains $\Omega_{h}$,

$$
\begin{aligned}
& \begin{aligned}
\widetilde{V}_{h}:=\left\{\widetilde{v}_{h} \in \mathcal{C}^{0}\left(\bar{\Omega}_{h}\right) \mid \widetilde{v}_{h_{T_{i}}}\right. & \text { is linear on every } T_{i} \in \mathcal{T}_{h} \text { and } \\
& \left.\widetilde{v}_{h}^{+}-\widetilde{v}_{h}^{-}=\beta \text { on } \Sigma \text { with any } \beta \in \mathbb{R}\right\}
\end{aligned} \\
& \widetilde{V}_{h}^{0}:=\left\{\widetilde{v}_{h} \in \widetilde{V}_{h} \mid \widetilde{v}_{h}^{+}-\widetilde{v}_{h}^{-}=0 \quad \text { on } \Sigma\right\},
\end{aligned}
$$

$$
\widetilde{H}_{h}:=\left\{\widetilde{\psi}_{h} \in L_{2}\left(\Gamma_{\infty}^{h}\right) \mid \widetilde{\psi}_{h_{S_{i}}} \text { is constant on every } S_{i} \in \mathcal{S}_{h} \text { and }\left\langle\widetilde{\psi}_{h}, 1\right\rangle_{h}=0\right\}
$$

and the set of admissible finite elements

$$
\widetilde{K}_{s_{0}}:=\left\{\left.\widetilde{v}_{h} \in \widetilde{V}_{h}|| \nabla \widetilde{v}_{h}\right|^{2} \leq s_{0}\right\} .
$$


Here we use the notation

$$
\langle\phi, \psi\rangle_{h}:=\int_{\Gamma_{\infty}^{h}} \phi(s) \psi(s) d s
$$

for the $L_{2}$-scalar product on $\Gamma_{\infty}^{h}$.

For the following, we need an approximate kernel $K_{h}$ which is defined as in (1.24) where $\vec{n}(\vec{y})$ is to be replaced by $\vec{n}_{h}(\vec{y})$, the linear interpolant of the normal vectors to $\Gamma_{\infty}$ at the vertices of $\Gamma_{\infty}^{h}$. The associated operator will be denoted by $\mathcal{K}_{h}$. We further define the discrete forms

$$
\begin{aligned}
a_{h}(\widetilde{u} \mid \widetilde{v}, \widetilde{w}) & :=\int_{\Omega_{h}} \rho\left(|\nabla \widetilde{u}|^{2}\right) \nabla \widetilde{v} \cdot \nabla \widetilde{w} d x, \\
\widetilde{b}_{h}(\widetilde{\lambda}, \widetilde{\psi}) & :=\langle\mathcal{V} \widetilde{\lambda}, \widetilde{\psi}\rangle_{h}, \\
\widetilde{d}_{h}(\widetilde{u}, \widetilde{\psi}) & :=\left\langle\mathcal{K}_{h} \widetilde{u}, \widetilde{\psi}\right\rangle_{h}, \\
\widetilde{\ell}_{1}^{h}(\widetilde{v}, \beta) & :=\rho_{\infty}\left\langle\nabla \Psi(\cdot, \beta) \cdot \vec{n}_{h}, \widetilde{v}\right\rangle_{h}, \\
\widetilde{\ell}_{2}^{h}(\widetilde{\psi}, \beta) & :=\left\langle\left(\mathcal{I}-\mathcal{K}_{h}\right) \Psi(\cdot, \beta), \widetilde{\psi}\right\rangle_{h} .
\end{aligned}
$$

The function $\Psi$ was defined in (1.10). Now the discrete analogue of problem (1.37) reads as follows:

\section{The discrete variational problem}

Find $\left(\widetilde{u}_{h}, \widetilde{\lambda}_{h}, \beta_{h}\right) \in \widetilde{K}_{s_{0}} \times \widetilde{H}_{h} \times \mathbb{R} \times \widetilde{H}_{h} \times \mathbb{R}$ such that

$$
\begin{array}{lll}
a_{h}\left(\widetilde{u}_{h} \mid \widetilde{u}_{h}, \widetilde{v}_{h}\right)-\left\langle\widetilde{\lambda}_{h}, \widetilde{v}_{h}\right\rangle_{h} & =\widetilde{\ell}_{1}^{h}\left(\widetilde{v}_{h}, \beta_{h}\right) & \text { for all } \widetilde{v}_{h} \in \widetilde{V}_{h}, \\
\widetilde{b}_{h}\left(\widetilde{\lambda}_{h}, \widetilde{\psi}_{h}\right)+\left\langle\widetilde{u}_{h}, \widetilde{\psi}_{h}\right\rangle_{h}-\widetilde{d}_{h}\left(\widetilde{u}_{h}, \widetilde{\psi}_{h}\right) & =\widetilde{\ell}_{2}^{h}\left(\widetilde{\psi}_{h}, \beta_{h}\right) & \text { for all } \widetilde{\psi}_{h} \in \widetilde{H}_{h}
\end{array}
$$

subject to $F\left(\beta_{h}\right)=0$.

Problem (3.7) leads to a system of nonlinear equations, where we have one unknown per node in the triangulation $\mathcal{T}_{h}$ of $\Omega_{h}$, one unknown per segment $S_{i}$ of the polygonal boundary $\Gamma_{\infty}^{h}$ and the unknown circulation $\beta_{h}$.

For the error analysis below we convert problem (3.7) into a form which allows the use of subspaces to the admissible function spaces used in (1.37). We will define subspaces $V_{h}, V_{h}^{0}$ and $H_{h}$ satisfying the conformity inclusions $V_{h} \subset V, V_{h}^{0} \subset V^{0}$ and $H_{h} \subset H$. This reformulation of problem (3.7) will enable us to present an analysis similar to that for conforming finite and boundary elements, see Johnson and Nedelec [39]. We introduce a mapping $\Phi_{h}: \Gamma_{\infty}^{h} \rightarrow \Gamma_{\infty}$, where $\Phi_{h}(x)$ is the point on $\Gamma_{\infty}$ closest to the point $x \in \Gamma_{\infty}^{h}$. For $h$ sufficiently small, the mapping $\Phi_{h}$ becomes a bijection, see LeRoux [43]. Hence, the inverse mapping $\Phi_{h}^{-1}$ exists. It transforms integrals along $\Gamma_{\infty}^{h}$ into integrals along $\Gamma_{\infty}$ by

$$
\int_{\Gamma_{\infty}^{h}} \widetilde{\psi}(s) d s=\int_{\Gamma_{\infty}} \widetilde{\psi} \circ \Phi_{h}^{-1}(s) J\left(\Phi_{h}^{-1}(s)\right) d s,
$$

where $J\left(\Phi_{h}^{-1}\right)=\left|\frac{\partial \Phi_{h}^{-1}}{\partial s}\right|$ is the one-dimensional Jacobian. Here $\frac{\partial}{\partial s}$ denotes the differentiation in the tangential direction.

We now define the conforming boundary space

$$
H_{h}:=\left\{\psi=J\left(\Phi_{h}^{-1}\right) \widetilde{\psi}_{h} \circ \Phi_{h}^{-1} \mid \widetilde{\psi}_{h} \in \widetilde{H}_{h}\right\} \subset H
$$


We also need an appropriate subspace $V_{h} \subset V$ based on the definition of $\widetilde{V}_{h}$. Let $\widetilde{v}_{h} \in \widetilde{V}_{h}$ be an arbitrarily given function. Then $\widetilde{v}_{h}$ is well-defined in $\Omega \cap \Omega_{h}$ by taking the restriction. In the skin

$$
\omega_{\infty}^{h}:=\left(\Omega \backslash \Omega_{h}\right) \cap\left\{x \mid \operatorname{dist}\left(x, \Gamma_{\infty}\right) \leq h\right\},
$$

however, we need an extension of $\widetilde{v}_{h}$ which we define by setting

$$
v_{h}(y):=\widetilde{v}_{h}(x)
$$

with $x \in \Gamma_{\infty}^{h}$ and any given $y$ lying on the line segment between the two points $x \in \Gamma_{\infty}^{h}$ and $\Phi_{h}(x) \in \Gamma_{\infty}$. In the same way as described above, we define the function $\widetilde{v}_{h}$ in the skin

$$
\omega_{P}^{h}=\left(\Omega \backslash \Omega_{h}\right) \cap\left\{x \mid \operatorname{dist}\left(x, \Gamma_{P}\right) \leq h\right\} .
$$

This defines an extension operator which is only slightly different from Zlamal's operator used by Feistauer and Ženíšek [32]. Our version is chosen in accordance with the boundary element approximation defined by LeRoux in [43] and used by Johnson and Nedelec [39]. Note that for the piecewise $\mathcal{C}^{\infty}$-curve $\Gamma_{P}$ we have

$$
\operatorname{meas}\left\{\left(\operatorname{supp} w_{h_{i}}\right) \cap \omega_{P}^{h}\right\} \leq c h_{i}^{3} \leq h_{i} A_{i} .
$$

We now denote by $V_{h}$ the space of all functions $v_{h}$ defined from $\widetilde{v}_{h} \in \widetilde{V}_{h}$ in the above way. Since on $\Omega_{h} \backslash \Omega$ the functions $v_{h}$ are well defined, $V_{h}$ consists of functions given on $\Omega \cup \Omega_{h}$ while their restrictions to $\Omega$ define a subspace of $V$. By $V_{h}^{0}$ we denote the set of all functions $v_{h} \in V_{h}$, which are continuous across the slit $\Sigma$. By changing the integrations from $\Gamma_{\infty}^{h}$ to $\Gamma_{\infty}$ and using the definitions of $V_{h}, V_{h}^{0}$ and $H_{h}$, we can reformulate problem (3.7) as follows. We note that

$$
a_{h}\left(u_{h} \mid v_{h}, w_{h}\right):=a_{h}\left(\widetilde{u}_{h} \mid \widetilde{v}_{h}, \widetilde{w}_{h}\right)
$$

and define

$$
\begin{aligned}
b_{h}(\lambda, \psi) & :=\frac{2}{\rho\left(\left|\vec{v}_{\infty}\right|^{2}\right) \sqrt{1-M_{\infty}^{2}}} \int_{\Gamma_{\infty} \Gamma_{\infty}} \lambda(y) \psi(x) G\left(\Phi_{h}^{-1}(x), \Phi_{h}^{-1}(y)\right) d s_{x} d s_{y}, \\
d_{h}(u, \psi) & :=\frac{2}{\sqrt{1-M_{\infty}^{2}}} \iint_{\Gamma_{\infty} \Gamma_{\infty}} u(y) \psi(x) \cdot K\left(\Phi_{h}^{-1}(x), \Phi_{h}^{-1}(y)\right)\left[J \circ\left(\Phi_{h}^{-1}(y)\right] d s_{x} d s_{y},\right. \\
\ell_{1}^{h}(v, \beta) & :=\rho_{\infty}\left\langle\left(\nabla \Psi(\cdot, \beta) \cdot \vec{n}_{h}\right) \circ \Phi_{h}^{-1}, v\right\rangle, \\
\ell_{2}^{h}(\psi, \beta) & :=\left\langle\Psi(\cdot, \beta) \circ \Phi_{h}^{-1}, \psi\right\rangle-d_{h}\left(\Psi(\cdot, \beta) \circ \Phi_{h}^{-1}, \psi\right) .
\end{aligned}
$$

Note that the transformations $\Phi_{h}$ do not always map all functions of $\widetilde{K}_{s_{0}}$ into $K_{s_{0}}$. But taking $s_{0}^{\prime}<s_{0}$, the functions on $\widetilde{K}_{s_{0}^{\prime}}$ are mapped into $K_{s_{0}}$ provided $h>0$ is small enough. This distinction of $s_{0}^{\prime}$ and $s_{0}$, however, is not significant for our error analysis. Therefore, in the following we carry out the analysis for the admissible approximations in $V_{h} \cap K_{s_{0}}$. Using these definitions we obtain:

The conforming discrete variational problem. Find $\left(u_{h}, \lambda_{h}, \beta_{h}\right) \in\left(V_{h} \cap\right.$ $\left.K_{s_{0}}\right) \times H_{h} \times \mathbb{R}$ such that

$$
\begin{array}{lll}
a_{h}\left(u_{h} \mid u_{h}, v_{h}\right)-\left\langle\lambda_{h}, v_{h}\right\rangle & =\ell_{1}^{h}\left(v_{h}, \beta_{h}\right) & \text { for all } v_{h} \in V_{h}^{0} \quad \text { and } \\
b_{h}\left(\lambda_{h}, \psi_{h}\right)+\left\langle u_{h}, \psi_{h}\right\rangle-d_{h}\left(u_{h}, \psi_{h}\right) & =\ell_{2}^{h}\left(\psi_{h}, \beta_{h}\right) & \text { for all } \psi_{h} \in H_{h}
\end{array}
$$

subject to $F\left(\beta_{h}\right)=0$. 
The following consistency estimates essentially follow from the corresponding results by Feistauer and Ženíšek [32] and by Johnson and Nedelec [39], respectively.

Lemma 3.1. Provided the polygonal approximation $\mathcal{S}_{h}$ of $\Gamma_{\infty}$ satisfies an inverse assumption, we have the following estimates for the bilinear and linear forms (3.10):

$$
\begin{gathered}
\left|a\left(u_{h} \mid u_{h}, v_{h}\right)-a_{h}\left(u_{h} \mid u_{h}, v_{h}\right)\right| \leq \operatorname{ch}\left\|u_{h}\right\|_{W^{1,2}(\Omega)}\left\|v_{h}\right\|_{W^{1,2}(\Omega)} \\
\qquad \text { for all }\left(u_{h}, v_{h}\right) \in\left(V_{h} \cap K_{s_{0}}\right) \times V_{h}, \\
\left|b\left(\lambda_{h}, \psi_{h}\right)-b_{h}\left(\lambda_{h}, \psi_{h}\right)\right| \leq \operatorname{ch}\left\|\lambda_{h}\right\|_{H^{-\frac{1}{2}}\left(\Gamma_{\infty}\right)}\left\|\psi_{h}\right\|_{H^{\frac{1}{2}}\left(\Gamma_{\infty}\right)} \\
\qquad \text { for all }\left(\lambda_{h}, \psi_{h}\right) \in H_{h} \times H_{h}, \\
\left|d\left(v, \psi_{h}\right)-d_{h}\left(v, \psi_{h}\right)\right| \leq \quad h^{3 / 2}\|v\|_{W^{1,2}(\Omega)}\left\|\psi_{h}\right\|_{H^{-\frac{1}{2}}\left(\Gamma_{\infty}\right)} \\
\quad \text { for all }(v, \psi) \in V \times H_{h}, \\
\left|\ell_{1}^{h}(v, \beta)-\ell_{1}(v, \beta)\right| \leq\left(c_{1}+c_{2}|\beta|\right) h\|v\|_{W^{1,2}(\Omega)} \quad \text { for all } v \in V, \\
\left|\ell_{2}^{h}(\psi, \beta)-\ell_{2}(\psi, \beta)\right| \leq\left(c_{1}+c_{2}|\beta|\right) h\|\psi\|_{H^{-\frac{1}{2}}\left(\Gamma_{\infty}\right)} \text { for all } \psi \in H .
\end{gathered}
$$

Proof. The estimates (3.13) and (3.14) can be found in the paper by Johnson and Nedelec [39]. Note that we need the inverse assumption for $\widetilde{H}_{h}$ associated with $\left\{\mathcal{S}_{h}\right\}_{h>0}$ for the proofs of (3.13), (3.14). The proof of (3.12) is due to the fact that on the skin the inequality

$$
\left\|v_{h}\right\|_{W^{1,2}\left(\omega_{\infty}^{h} \cup \omega_{P}^{h}\right)} \leq c h^{\frac{1}{2}}\left\|v_{h}\right\|_{W^{1,2}(\Omega)} \quad \text { for all } v_{h} \in V_{h}
$$

holds. This inequality can be found in [32, Lemma 3.3.12], however for a slightly different extension operator. But the proof in our case is completely analogous to the one by Feistauer and Ženížek in [32]. Thus we obtain

$$
\begin{aligned}
\left|a\left(u_{h} \mid u_{h}, v_{h}\right)-a_{h}\left(u_{h} \mid u_{h}, v_{h}\right)\right| & \leq \int_{\omega_{\infty}^{h} \cup \omega_{P}^{h}} \rho\left(\left|\nabla u_{h}\right|^{2}\right)\left|\nabla u_{h}\right|\left|\nabla v_{h}\right| d x \\
& \leq \rho_{0}\left\|u_{h}\right\|_{W^{1,2}\left(\omega_{\infty}^{h} \cup \omega_{P}^{h}\right)}\left\|v_{h}\right\|_{W^{1,2}\left(\omega_{\infty}^{h} \cup \omega_{P}^{h}\right)} \\
& \leq \operatorname{ch}\left\|u_{h}\right\|_{W^{1,2}(\Omega)}\left\|v_{h}\right\|_{W^{1,2}(\Omega)} .
\end{aligned}
$$

The functions $\left(\nabla \Psi \cdot \vec{n}_{h}\right) \circ \Phi_{h}^{-1}$ and $\Psi \circ \Phi_{h}^{-1}$ are piecewise uniformly Lipschitz continuous and their derivatives are uniformly bounded in $\omega_{\infty}^{h}$. Hence we get the estimates

$$
\left\{\begin{aligned}
\left|\left[\left(\nabla \Psi \cdot \vec{n}_{h}\right) \circ \Phi_{h}^{-1}-\nabla \Psi \cdot \vec{n}\right](x, \beta)\right| & \leq\left(c_{1}+c_{2}|\beta|\right) h \\
\left|\left[\Psi \circ \Phi_{h}^{-1}-\Psi\right](x, \beta)\right| & \leq\left(c_{1}+c_{2}|\beta|\right) h
\end{aligned} \quad \text { for all } x \in \Gamma_{\infty} .\right.
$$

Thus, with (3.14) and (3.16) we obtain

$$
\begin{aligned}
\left|\ell_{1}^{h}(v, \beta)-\ell_{1}(v, \beta)\right| & =\rho_{\infty}\left|\left\langle\left[\left(\nabla \Psi \cdot \vec{n}_{h}\right) \circ \Phi_{h}^{-1}-(\nabla \Psi \cdot \vec{n})\right], v\right\rangle\right| \\
& \leq\left(c_{1}+c_{2}|\beta|\right) h\|v\|_{L_{1}\left(\Gamma_{\infty}\right)} \\
& \leq\left(c_{1}+c_{2}|\beta|\right) h\|v\|_{W^{1,2}(\Omega)} \text { for all } v \in V
\end{aligned}
$$

the first of the desired estimates (3.15). Similarly, for the second estimate of (3.15) we find with(3.14) 


$$
\begin{aligned}
\left|\ell_{2}^{h}(\psi, \beta)-\ell_{2}(\psi, \beta)\right| \leq & \left|\left\langle\Psi \circ \Phi_{h}^{-1}-\Psi, \psi\right\rangle\right|+\left|d_{h}\left(\Psi \circ \Phi_{h}^{-1}, \psi\right)-d(\Psi, \psi)\right| \\
\leq & \left(c_{1}+c_{2}|\beta|\right) h\|\psi\|_{H^{-1 / 2}\left(\Gamma_{\infty}\right)} \\
& +\left|d_{h}\left(\Psi \circ \Phi_{h}^{-1}-\Psi, \psi\right)\right|+\left|d_{h}(\Psi, \psi)-d(\Psi, \psi)\right| \\
\leq & \left(c_{1}+c_{2}|\beta|\right) h\|\psi\|_{H^{-1 / 2}\left(\Gamma_{\infty}\right)} \cdot \square
\end{aligned}
$$

For later use we collect the following finite element approximation results.

Lemma 3.2. (a) Let us denote by $P_{h}: H \rightarrow H_{h}$ the $L^{2}$-projection defined by

$$
\left\langle P_{h} \varphi, \psi_{h}\right\rangle:=\left\langle\varphi, \psi_{h}\right\rangle \quad \text { for all } \psi_{h} \in H_{h} .
$$

Then we have for every $\varphi \in H$

$$
\left\|\varphi-P_{h} \varphi\right\|_{H^{-\frac{1}{2}}\left(\Gamma_{\infty}\right)} \rightarrow 0 \text { for } h \rightarrow 0 .
$$

(b) Further, we define the Ritz projections $\widetilde{R}_{h}: V \rightarrow \widetilde{V}_{h}$ resp. $R_{h}: V \rightarrow V_{h}$ by

$$
\int_{\Omega_{h}} \nabla \widetilde{R}_{h} v \cdot \nabla \widetilde{v}_{h} d x=\int_{\Omega} \nabla v \cdot \nabla v_{h} d x \quad \text { for all } \widetilde{v}_{h} \in \widetilde{V}_{h}
$$

where $v_{h} \in V_{h}$ is the extension of $\widetilde{v}_{h}$ to $\Omega$. Then

$$
\lim _{h \rightarrow 0}\left\|v-\widetilde{R}_{h} v\right\|_{W^{1,2}(\Omega)}=0 \quad \text { for all } v \in V
$$

and

$$
\left\|\widetilde{R}_{h} v\right\|_{W^{1,2}\left(\Omega_{h}\right)} \leq c\|v\|_{W^{1,2}(\Omega)} \quad \text { for all } v \in V .
$$

(c) There exist families $\left\{\mathcal{T}_{h}\right\}_{h>0}$ of triangulations having additional properties which imply that the Ritz projection defined in (3.19) satisfies the stability estimate

$$
\left\|\nabla \widetilde{R}_{h} v\right\|_{L^{\infty}\left(\Omega_{h}\right)} \leq c^{*}\|\nabla v\|_{L^{\infty}(\Omega)} \quad \text { for all } v \in W^{1, \infty}(\Omega)
$$

where the constant $c^{*} \geq 1$ is independent of $v, v_{h}$ and $h$.

Proof. (a) and (b) are well known properties, see Babuška and Aziz [3]. The estimate (3.20) in (c) is e. g. a consequence of the quasiuniformity assumption, i. e. the angle property is satisfied and each triangle $T_{i} \in \mathcal{T}_{h}$ contains a circle of radius $c h$ where the constant $c>0$ does not depend on $T_{i}$ or $h$, as was proved by Rannacher and Scott [52]. For the Ritz projection associated with homogeneous Dirichlet conditions, Crouzeix and Thomée [25] prove (3.20) for a much wider class of families $\left\{\mathcal{T}_{h}\right\}_{h>0}$ which includes grids generated by most adaptive methods. Under our assumptions on $\Gamma_{P}, \Sigma$ and $\Gamma_{\infty}$, their proof can be modified so that (c) also holds for the Ritz projection (3.19).

\section{The DisCREte Minimization PROBLEM}

The goal of this section is the formulation of problem (3.7) as a discrete minimization problem. The underlying idea goes back to Glowinski and Pironneau [36], and has since been further developed, see Bristeau et al. [15] or Berger et al. [9]. For 
transonic flow, the hyperbolic character of the supersonic region creates additional difficulties. As pointed out in Section 2, we must take into account an additional selection principle. This will be done by a penalization due to Glowinski and Pironneau [36]. To this end, we define the following functional $\mathcal{J}_{h}: \widetilde{V}_{h} \cap \widetilde{K}_{s_{0}} \rightarrow \mathbb{R}$ by

$$
\mathcal{J}_{h}\left(\phi_{h}\right):= \begin{cases}\frac{1}{2} \int_{\Omega_{h}}\left|\nabla \xi_{h}\left(\phi_{h}\right)\right|^{2} d x & \text { if globally } s_{0}<\frac{2 a_{0}^{2}}{\kappa+1} \text { (subsonic flow) } \\ \frac{1}{2} \int_{\Omega_{h}}\left|\nabla \xi_{h}\left(\phi_{h}\right)\right|^{2} d x+\mathcal{P}_{h}\left(\phi_{h}\right) & \text { for } s_{0}<\frac{2 a_{0}^{2}}{\kappa-1} \text { (transonic flow) }\end{cases}
$$

where the penalty functional $\mathcal{P}_{h}: \widetilde{V}_{h} \cap \widetilde{K}_{s_{0}} \rightarrow \mathbb{R}$ is given by

$$
\mathcal{P}_{h}\left(\phi_{h}\right):=\frac{\mu}{2} \sum_{\substack{i=1 \\ p_{i} \notin \Sigma \cup \Gamma \infty}}^{N} \frac{1}{A_{i}^{\epsilon}}\left(\left[-\int_{\Omega_{h}} \nabla \phi_{h} \cdot \nabla w_{h_{i}} d x-B \int_{\Omega_{h}} w_{h_{i}} d x\right]^{+}\right)^{2} .
$$

By $[.]^{+}$we denote the nonnegative part of the quantity in brackets. Here, $\mu>0$, $B>0$ and $2>\epsilon>1$ are constants, which do not depend on $h$. These constants can be chosen according to numerical experiments depending on the specific profile, the travelling velocity $\vec{v}_{\infty}$ etc., but then they are fixed for mesh refinement. The function $\xi_{h}\left(\phi_{h}\right) \in \widetilde{V}_{h}^{0}$ is the solution of the following state equation

$$
\begin{aligned}
\int_{\Omega_{h}} & \nabla \xi_{h}\left(\phi_{h}\right) \cdot \nabla v_{h} d x \\
\quad & =a_{h}\left(\phi_{h} \mid \phi_{h}, v_{h}\right)-\left\langle\lambda_{h}\left(\phi_{h}, \beta_{h}\right), v_{h}\right\rangle_{h}-\widetilde{\ell}_{1}^{h}\left(v_{h}, \beta_{h}\right) \text { for all } v_{h} \in \widetilde{V}_{h}^{0} .
\end{aligned}
$$

This state equation is the finite element approximation of the Neumann problem for the Poisson equation with given Neumann data on $\Gamma_{P}$, see (1.15), and on $\Gamma_{\infty}$ where $\lambda_{h}$ is given. On the other hand, $\lambda_{h}\left(\varphi_{h}\right) \in \widetilde{H}_{h}$ is to be determined by the Galerkin discretization of the boundary integral equation (1.28), i.e.

$$
\widetilde{b}_{h}\left(\lambda_{h}\left(\phi_{h}, \beta_{h}\right), \psi_{h}\right)=\widetilde{\ell}_{2}^{h}\left(\psi_{h}, \beta_{h}\right)-\left\langle\phi_{h}, \psi_{h}\right\rangle_{h}+\widetilde{d}_{h}\left(\phi_{h}, \psi_{h}\right) \text { for all } \psi_{h} \in \widetilde{H}_{h} .
$$

Instead of solving the discrete equations (3.7), we will now solve the following:

\section{Discrete minimization problem}

Find $\left(u_{h}, \lambda_{h}, \beta_{h}\right) \in \widetilde{K}_{s_{0}} \times \widetilde{H}_{h} \times I$ such that

$$
\mathcal{J}_{h}\left(u_{h}\right):=\min _{\phi_{h} \in \widetilde{K}_{s_{0}}} \mathcal{J}_{h}\left(\phi_{h}\right)
$$

under the constraints (2.4) and $F\left(\beta_{h}\right)=0$ where $\mathcal{J}_{h}\left(\Phi_{h}\right)$ is defined via (4.1) and $I \subset \mathbb{R}$ is an appropriately fixed finite interval.

Remark. To simplify the notation, we are not using the ${ }^{\sim}$-sign for finite element functions as previously.

Since $\beta_{h}$ is the circulation of $u_{h}$, it is sought only in a bounded interval $I$. The solution of problem (4.5) subject to $F\left(\beta_{h}\right)=0$, to (4.3) and (4.4) exists, because we 
minimize a differentiable functional over a bounded, convex and nonempty subset of a finite-dimensional space. The nonemptyness is due to the fact that the zero function lies in $\widetilde{K}_{s_{0}}$ and satisfies $F(0)=0$. Note that the minimization of the first term of (4.1) in view of (4.3) is equivalent to the minimization of the $W^{1,2}(\Omega)$ seminorm of the Riesz representation of the residual of (1.3) in the least squares sense. The above method, hence, can be considered as a least squares method.

The solution $u_{h} \in \widetilde{K}_{s_{0}}$ is not necessarily unique; nevertheless, any solution $u_{h}$ defines a corresponding flux $\lambda_{h}\left(u_{h}\right) \in \widetilde{H}_{h}$ as the unique solution of (4.4). Thus we may assume for a given sequence of meshsizes the existence of a sequence $\left\{\left(u_{h}, \lambda_{h}, \beta_{h}\right)\right\}_{h>0}$, where $u_{h} \in \widetilde{K}_{s_{0}}$ is a solution of (4.5) and $\lambda_{h} \in \widetilde{H}_{h}$ is the corresponding solution of (4.4) with the corresponding $\beta_{h}$. If $\mathcal{J}_{h}\left(u_{h}\right)=0$, then $\left(u_{h}, \lambda_{h}, \beta_{h}\right)$ is a solution of (3.7).

The discrete minimization problem (4.5) can be solved by a Polak-Ribière type conjugate gradient algorithm, which takes into account the constraint $F(\beta)=0$ and the weak coupling equation (4.4). The method we used is described in detail in $[10]$.

\section{ON THE CONVERGENCE OF THE MINIMIZATION METHOD}

The goal of this section is the convergence proof for the sequence $\left\{\left(\widetilde{u}_{h}, \tilde{\lambda}_{h}, \beta_{h}\right)\right\}_{h>0}$ of solutions of the minimization problems (4.5). Under the assumption of existence and uniqueness for the solution to problem (1.37) we will show that the sequence

$$
\left\{\left(\widetilde{u}_{h}, \tilde{\lambda}_{h}, \beta_{h}\right)\right\}_{h>0} \in \widetilde{K}_{s_{0}} \times \widetilde{H}_{h} \times I
$$

converges to this solution. For the case of subsonic flow, the proof of this assertion is straightforward. The case of transonic flow will be more difficult. For the proof we need a compactness result from Mandel and Nečas [44] and Murat [49]. For this case, a discrete entropy condition will play the crucial role and will enforce the convergence.

5.1. The case of subsonic flow. For this case, we need first a preliminary result which involves standard results of approximation theory.

Lemma 5.1. Let $u \in V \cap K_{s_{0}}$ with $s_{0}<\frac{2 a_{0}^{2}}{\kappa+1}$ be a given function which has the additional regularity property

$$
u \in W^{2+\varepsilon, 2}(\Omega) \text { for some } \varepsilon>0 .
$$

To the function $u$ there corresponds a $\beta \in \mathbb{R}$ which satisfies $F(\beta)=0$. Then for sufficiently small meshsizes $h$, there exists a sequence of functions $\left\{\widetilde{u}_{h}^{I}\right\}_{h>0}$ with corresponding $\widetilde{\beta}_{h}$ satisfying

$$
\begin{aligned}
& \widetilde{u}_{h}^{I} \in \widetilde{V}_{h} \cap \widetilde{K}_{\widetilde{s}_{0}} \text { with } s_{0}<\widetilde{s}_{0}<\frac{2 a_{0}^{2}}{\kappa+1}, \\
& F\left(\widetilde{\beta_{h}}\right):=\left.\left|\nabla \widetilde{u}_{h}^{I}\right|_{T_{+}} \cdot \vec{t}_{+}\right|^{2}-\left.\left|\nabla \widetilde{u}_{h}^{I}\right|_{T_{-}} \cdot \vec{t}_{-}\right|^{2}=0, \\
& \left\|u-u_{h}^{I}\right\|_{W^{1, p}(\Omega)} \stackrel{h \rightarrow 0}{\longrightarrow} 0 \quad \text { for every } p \in[1, \infty] .
\end{aligned}
$$




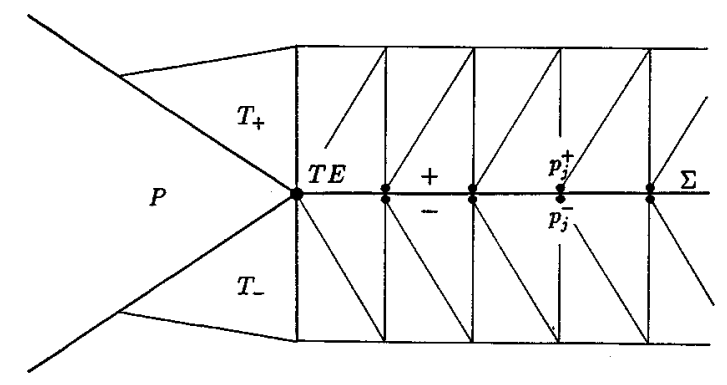

FIGURE 2. The triangular grid involving the Kutta-Joukowski condition

Here $u_{h}^{I} \in V_{h}$ is the extension of $\widetilde{u}_{h}^{I} \in \widetilde{V}_{h}$ to all of $\Omega$ as defined in Section 3. $\vec{t}_{+}, \vec{t}_{-}$are the unit tangential vectors at the trailing edge pointing in the direction of TE. Let $T_{+}$and $T_{-}$denote the respective triangles adjacent to TE as indicated in Figure 2.

Proof. Let us denote by $\widetilde{\Pi}_{h} u$ the interpolant of $u$ in $\Omega_{h}$. Since $u \in V$ we have $\widetilde{\Pi}_{h} u \in \widetilde{V}_{h}$. By $\Pi_{h} u$ we denote the extension of $\widetilde{\Pi}_{h} u$ into $V_{h}$. Using (5.1), standard approximation results for $\varepsilon \in \mathbb{N}_{0}$, see Ciarlet [18, p. 123], interpolating these inequalities for noninteger $\varepsilon$ and finally using the property that the width of the skin is of order $h^{2}$, we obtain

$$
\left\|u-\Pi_{h} u\right\|_{W^{1, \infty}(\Omega)} \leq \operatorname{ch}^{\varepsilon}\|u\|_{W^{2+\varepsilon, 2}(\Omega)} .
$$

The definition of $\Pi_{h} u$ implies that on $\Sigma$ the equation $\left(\Pi_{h} u\right)^{+}-\left(\Pi_{h} u\right)^{-}=\beta$ still holds.

Note that $\widetilde{\Pi}_{h} u$ does not satisfy the Kutta-Joukowski condition exactly, but only approximately; with (5.3), however, we obtain

$$
\left.\left|\nabla \widetilde{\Pi}_{h} u\right|_{T_{+}} \cdot \vec{t}_{+}\right|^{2}-\left.\left|\nabla \widetilde{\Pi}_{h} u\right|_{T_{-}} \cdot \vec{t}_{-}\right|^{2}=O\left(h^{\varepsilon}\right) .
$$

We shall now modify the interpolant $\widetilde{\Pi}_{h} u$ along the slit $\Sigma$ by changing the function values only in the respective upper and lower points $p_{j}^{+}$and $p_{j}^{-}$on $\Sigma$ as described in [10]. The modified function will be denoted by $\widetilde{u}_{h}^{I} \in \widetilde{V}_{h}$ and it satisfies $F\left(\widetilde{\beta}_{h}\right)=0$ with a corresponding $\widetilde{\beta}_{h} \in \mathbb{R}$. From the construction of $\widetilde{u}_{h}^{I}$ it follows that the inequalities

$$
\left|\beta-\widetilde{\beta}_{h}\right| \leq \operatorname{ch}^{1+\varepsilon}
$$

and

$$
\left\|\widetilde{\Pi}_{h} u-\widetilde{u}_{h}^{I}\right\|_{W^{1, \infty}\left(\Omega^{h}\right)} \leq c h^{\varepsilon / 2}
$$

are valid. Thus, combining (5.3) and (5.6), we obtain

$$
\left\|u-u_{h}^{I}\right\|_{W^{1, p}(\Omega)} \stackrel{h \rightarrow 0}{\longrightarrow} 0 \text { for all } p \in[1, \infty]
$$


Now since $s_{0}<\frac{2 a_{0}^{2}}{\kappa+1}$, for sufficiently small $h$ we can find a constant $\widetilde{s}_{0}<\frac{2 a_{0}^{2}}{\kappa+1}$ such that $\left|\nabla u_{h}^{I}\right|^{2} \leq \widetilde{s}_{0}$ holds a.e. in $\Omega$.

We shall now prove the convergence in $V \times H \times I$ of the sequence $\left\{\left(\widetilde{u}_{h}, \widetilde{\lambda}_{h}, \beta_{h}\right)\right\}_{h>0}$ of solutions of the minimization problems (4.5). Again, we shall denote by $\left\{\left(u_{h}, \lambda_{h}, \beta_{h}\right)\right\}_{h>0}$ the corresponding sequence in $\left(V_{h} \times H_{h} \times I\right)$.

Theorem 5.2. Let $u \in V \cap K_{s_{0}}$ with $s_{0}<\frac{2 a_{0}^{2}}{\kappa+1}, \lambda \in H$ and $\beta \in I$ be the unique solution of the coupled flow problem (1.37). Moreover let this solution possess the regularity $u \in W^{2+\varepsilon, 2}(\Omega)$ for some $\varepsilon>0$. Then there exists a constant $\widetilde{s}_{0}<\frac{2 a_{0}^{2}}{\kappa+1}$ such that the sequence $\left\{\left(\widetilde{u}_{h}, \widetilde{\lambda}_{h}, \beta_{h}\right)\right\}_{h>0}, \widetilde{u}_{h} \in \widetilde{K}_{\widetilde{s}_{0}}, \widetilde{\lambda}_{h} \in \widetilde{H}_{h}, \beta_{h} \in I$ of solutions of the minimization problem (4.5) converges in $V \times H \times I$ to the solution $(u, \lambda, \beta)$ of problem (1.37).

Proof. The proof of Theorem 5.2 will be split into several steps.

Step 1: Lemma 5.1 implies the existence of a constant $\widetilde{s}_{0}<\frac{2 a_{0}^{2}}{\kappa+1}$ and a function $\widetilde{u}_{h}^{I} \in \widetilde{V}_{h} \cap \widetilde{K}_{\widetilde{s}_{0}}$, satisfying (5.2). For this interpolant $\widetilde{u}_{h}^{I}$ we take $\beta \in \mathbb{R}$ corresponding to the exact solution and determine $\lambda_{h}^{I}$ by solving equation (4.4) with these $\widetilde{u}_{h}^{I}$ and $\beta$. By (4.3) we then determine the corresponding $\widetilde{\xi}_{h}$. Thus, using the definition of $\left\{\left(\widetilde{u}_{h}, \widetilde{\lambda}_{h}, \beta_{h}\right)\right\}_{h>0}$ as the solution of minimization problem (4.5) subject to $F\left(\beta_{h}\right)=$ 0 , we obtain

$$
0 \leq \mathcal{J}_{h}\left(\widetilde{u}_{h}\right)=\min _{\widetilde{\phi}_{h} \in \widetilde{K}_{\widetilde{s}_{0}}} \mathcal{J}_{h}\left(\widetilde{\phi}_{h}\right) \leq \mathcal{J}_{h}\left(\widetilde{u}_{h}^{I}\right)
$$

We will now consider the expression $\mathcal{J}_{h}\left(\widetilde{u}_{h}^{I}\right)$ as $h$ tends to zero. With definition (4.3), properties (1.31), (5.1), (5.3) and the interpolation property (5.4) we obtain

$$
\begin{aligned}
2 \mathcal{J}_{h}\left(\widetilde{u}_{h}^{I}\right)= & \int_{\Omega_{h}}\left|\nabla \widetilde{\xi}_{h}\left(\widetilde{u}_{h}^{I}\right)\right|^{2} d x \\
= & a_{h}\left(\widetilde{u}_{h}^{I} \mid \widetilde{u}_{h}^{I}, \widetilde{\xi}_{h}\right)-\left\langle\lambda_{h}^{I}, \widetilde{\xi}_{h}\right\rangle_{h}-\widetilde{\ell}_{1}^{h}\left(\widetilde{\xi}_{h}, \beta\right) \\
= & a_{h}\left(u_{h}^{I} \mid u_{h}^{I}, \xi_{h}\right)-\left\langle\lambda_{h}^{I}, \xi_{h}\right\rangle-\ell_{1}^{h}\left(\xi_{h}, \beta\right) \\
= & a_{h}\left(u_{h}^{I} \mid u_{h}^{I}, \xi_{h}\right)-a\left(u_{h}^{I} \mid u_{h}^{I}, \xi_{h}\right)+a\left(u_{h}^{I} \mid u_{h}^{I}, \xi_{h}\right)-a\left(u \mid u, \xi_{h}\right) \\
& +\left\langle\lambda-\lambda_{h}^{I}, \xi_{h}\right\rangle+\ell_{1}\left(\xi_{h}, \beta\right)-\ell_{1}^{h}\left(\xi_{h}, \beta\right) \\
\leq & c h\left\|u_{h}^{I}\right\|_{W^{1,2}(\Omega)}\left\|\xi_{h}\right\|_{W^{1,2}(\Omega)}+c\left\|u-u_{h}^{I}\right\|_{W^{1,2}(\Omega)}\left\|\xi_{h}\right\|_{W^{1,2}(\Omega)} \\
& +c\left\|\lambda-\lambda_{h}^{I}\right\|_{H^{-\frac{1}{2}}\left(\Gamma_{\infty}\right)}\left\|\xi_{h}\right\|_{H^{\frac{1}{2}}\left(\Gamma_{\infty}\right)}+\left(c_{1}+c_{2}|\beta|\right) h\left\|\xi_{h}\right\|_{W^{1,2}(\Omega)} \\
\leq & c\left\{h\left(\left\|u_{h}^{I}\right\|_{W^{1,2}(\Omega)}+1+|\beta|\right)\right. \\
\quad & \left.\quad+\left\|u-u_{h}^{I}\right\|_{W^{1,2}(\Omega)}+\left\|\lambda-\lambda_{h}^{I}\right\|_{H^{-\frac{1}{2}}\left(\Gamma_{\infty}\right)}\right\}\left\|\xi_{h}\right\|_{W^{1,2}(\Omega)} .
\end{aligned}
$$

Using the $L^{2}$-projection $P_{h}$ defined in Lemma 3.2, inequality (1.34), equations (1.37) and (3.11), the continuity of the form $d$ which follows from (1.29), together 
with the inequalities (1.33), (3.13), (3.14) and (3.15), we obtain the estimates

$$
\begin{array}{rl}
\gamma_{1}\left\|\lambda-\lambda_{h}^{I}\right\|_{H^{-\frac{1}{2}}\left(\Gamma_{\infty}\right) \leq}^{2} & b\left(\lambda-\lambda_{h}^{I}, \lambda-\lambda_{h}^{I}\right) \\
= & b\left(\lambda-\lambda_{h}^{I}, \lambda-P_{h} \lambda\right)+b\left(\lambda, P_{h} \lambda-\lambda_{h}^{I}\right)-b_{h}\left(\lambda_{h}^{I}, P_{h} \lambda-\lambda_{h}^{I}\right) \\
& +b_{h}\left(\lambda_{h}^{I}, P_{h} \lambda-\lambda_{h}^{I}\right)-b\left(\lambda_{h}^{I}, P_{h} \lambda-\lambda_{h}^{I}\right) \\
\leq & c\left\|\lambda-\lambda_{h}^{I}\right\|_{H^{-\frac{1}{2}}\left(\Gamma_{\infty}\right)}\left\|P_{h} \lambda-\lambda\right\|_{H^{-\frac{1}{2}}\left(\Gamma_{\infty}\right)} \\
& -\left\langle u, P_{h} \lambda-\lambda_{h}^{I}\right\rangle+d\left(u, P_{h} \lambda-\lambda_{h}^{I}\right)+\ell_{2}\left(P_{h} \lambda-\lambda_{h}^{I}, \beta\right) \\
& -d\left(u_{h}^{I}, P_{h} \lambda-\lambda_{h}^{I}\right)+d\left(u_{h}^{I}, P_{h} \lambda-\lambda_{h}^{I}\right) \\
& +\left\langle u_{h}^{I}, P_{h} \lambda-\lambda_{h}^{I}\right\rangle-d_{h}\left(u_{h}^{I}, P_{h} \lambda-\lambda_{h}^{I}\right)-\ell_{2}^{h}\left(P_{h} \lambda-\lambda_{h}^{I}, \beta\right) \\
& +c h\left\|\lambda_{h}^{I}\right\|_{H^{-\frac{1}{2}}\left(\Gamma_{\infty}\right)}\left\|P_{h} \lambda-\lambda_{h}^{I}\right\|_{H^{-\frac{1}{2}}\left(\Gamma_{\infty}\right)} \\
\leq & c\left\|\lambda-\lambda_{h}^{I}\right\|_{H^{-\frac{1}{2}}\left(\Gamma_{\infty}\right)}\left\|\lambda-P_{h} \lambda\right\|_{H^{-\frac{1}{2}}\left(\Gamma_{\infty}\right)}\left\|P_{h} \lambda-\lambda_{h}^{I}\right\|_{H^{-\frac{1}{2}}\left(\Gamma_{\infty}\right)} \\
& +c\left\|u-u_{h}^{I}\right\|_{H^{\frac{1}{2}}\left(\Gamma_{\infty}\right)}\left\|P_{h} \lambda-\lambda_{h}^{I}\right\|_{H^{-\frac{1}{2}}(\Gamma)} \\
& +c h^{\frac{3}{2}}\left\|u_{h}^{I}\right\|_{W^{1,2}(\Omega)} P_{h}^{I} \|_{H^{-\frac{1}{2}}\left(\Gamma_{\infty}\right)} \\
& +\left(c_{1}+c_{2}|\beta|\right) h\left\|P_{h} \lambda-\lambda_{h}^{I}\right\|_{h}\left\|P_{h}\right\|_{H^{-\frac{1}{2}}\left(\Gamma_{\infty}\right)}\left\|P_{h} \lambda-\lambda_{h}^{I}\right\|_{H^{-\frac{1}{2}}\left(\Gamma_{\infty}\right)}
\end{array}
$$

We use Young's inequality $a b \leq \varepsilon a^{2}+\frac{1}{4 \varepsilon} b^{2}$ and the triangle inequality to obtain with the appropriate choice of $\varepsilon$ the consistency estimate

$$
\begin{aligned}
\| \lambda- & \lambda_{h}^{I} \|_{H^{-\frac{1}{2}}\left(\Gamma_{\infty}\right)} \\
& \leq c\left\{\left\|\lambda-P_{h} \lambda\right\|_{H^{-\frac{1}{2}}\left(\Gamma_{\infty}\right)}+\left\|u-u_{h}^{I}\right\|_{W^{1,2}(\Omega)}+h\left\|\lambda_{h}^{I}\right\|_{H^{-\frac{1}{2}}\left(\Gamma_{\infty}\right)}\right\} .
\end{aligned}
$$

We remark that this estimate is also valid for boundary element collocation instead of the Galerkin method, see [2].

Then together with (5.8) and (5.9) we have

$$
\begin{aligned}
\mathcal{J}_{h}\left(\widetilde{u}_{h}^{I}\right) \leq c\{ & h\left(\left\|u_{h}^{I}\right\|_{W^{1,2}(\Omega)}+\left\|\lambda_{h}^{I}\right\|_{H^{-\frac{1}{2}}\left(\Gamma_{\infty}\right)}+1+|\beta|\right) \\
& \left.+\left\|\lambda-P_{h} \lambda\right\|_{H^{-\frac{1}{2}}\left(\Gamma_{\infty}\right)}+\left\|u-u_{h}^{I}\right\|_{W^{1,2}(\Omega)}\right\}\left\|\xi_{h}\right\|_{W^{1,2}(\Omega)} .
\end{aligned}
$$

The sequence $\left\{u_{h}^{I}\right\}_{h>0}$ is uniformly bounded in $W^{1,2}(\Omega)$ and hence by (3.11) and (1.34) we can show that $\left\{\lambda_{h}^{I}\right\}_{h>0}$ is also uniformly bounded in $H$. The construction of $\xi_{h} \in V_{h}^{0}$ from $\widetilde{\xi}_{h} \in \widetilde{V}_{h}^{0}$ implies together with (4.1) the estimate

$$
\left\|\xi_{h}\right\|_{W^{1,2}(\Omega)} \leq c\left\|\widetilde{\xi}_{h}\right\|_{W^{1,2}\left(\Omega_{h}\right)} \leq c\left\|\nabla \widetilde{\xi}_{h}\right\|_{L^{2}\left(\Omega_{h}\right)} \leq c \sqrt{\mathcal{J}_{h}\left(\widetilde{u}_{h}^{I}\right)} .
$$

Combining (5.7), (5.10), and (5.11), the boundedness of $\left\{u_{h}^{I}\right\}_{h>0}$ and $\left\{\lambda_{h}^{I}\right\}_{h>0}$ and the approximation properties of $u_{h}^{I}$ and $P_{h} \lambda$, we obtain

$$
0 \leq \mathcal{J}_{h}\left(\widetilde{u}_{h}\right) \leq \mathcal{J}_{h}\left(\widetilde{u}_{h}^{I}\right) \rightarrow 0 \quad \text { for } h \rightarrow 0 .
$$

2. Step: Since the sequence $\left\{u_{h}\right\}_{h>0} \subset K_{\widetilde{s}_{0}}$, it follows that the sequence is bounded in $V$. Again we can use (3.11) and the coercivity (1.34) to show that the sequence $\left\{\lambda_{h}\right\}_{h>0}$ is bounded in $H$. Therefore, the sequence $\left\{\left(u_{h}, \lambda_{h}, \beta_{h}\right)\right\}_{h>0}$ is 
bounded in $\left(V \cap K_{\widetilde{s}_{0}}\right) \times H \times I$. Since $V \cap K_{\widetilde{s}_{0}}$ is a closed convex subset of $V$ with respect to the topology in $V$, we may extract a subsequence $\left\{\left(u_{h}, \lambda_{h}, \beta_{h}\right)\right\}_{h>0}$ and find an element $(\hat{u}, \hat{\lambda}, \hat{\beta}) \in\left(V \cap K_{\widetilde{s}_{0}}\right) \times H \times I$ such that

$$
\begin{aligned}
& u_{h} \rightarrow \hat{u} \quad \text { weakly in } W^{1, p}(\Omega) \text { for every } p \in(2, \infty), \\
& \lambda_{h} \rightarrow \hat{\lambda} \quad \text { weakly in } H, \\
& \beta_{h} \rightarrow \hat{\beta} \quad \text { in } \mathbb{R} .
\end{aligned}
$$

We shall now show that $\left\{\left(u_{h}, \lambda_{h}, \beta_{h}\right)\right\}_{h>0}$ converges strongly to $(\hat{u}, \hat{\lambda}, \hat{\beta})$.

3. Step: We combine the inequalities (1.34) and (1.32) and obtain

$$
\begin{aligned}
\min \left(\gamma_{1}, \gamma_{2}\right)\left\{\left\|\hat{u}-u_{h}\right\|_{W^{1,2}(\Omega)}^{2}+\left\|\hat{\lambda}-\lambda_{h}\right\|_{H^{-\frac{1}{2}}\left(\Gamma_{\infty}\right)}^{2}\right\} \\
\leq \quad a\left(\hat{u} \mid \hat{u}, \hat{u}-u_{h}\right)-a\left(u_{h} \mid u_{h}, \hat{u}-u_{h}\right) \\
\quad+b\left(\hat{\lambda}, \hat{\lambda}-\lambda_{h}\right)-b\left(\lambda_{h}, \hat{\lambda}-\lambda_{h}\right) \\
=\quad a\left(\hat{u} \mid \hat{u}, \hat{u}-u_{h}\right)+b\left(\hat{\lambda}, \hat{\lambda}-\lambda_{h}\right) \\
-a\left(u_{h} \mid u_{h}, \hat{u}-\Pi_{h} \hat{u}\right)-b\left(\lambda_{h}, \hat{\lambda}-P_{h} \hat{\lambda}\right) \\
-a\left(u_{h} \mid u_{h}, \Pi_{h} \widehat{u}-u_{h}\right)+a_{h}\left(u_{h} \mid u_{h}, \Pi_{h} \widehat{u}-u_{h}\right) \\
-b\left(\lambda_{h}, P_{h} \hat{\lambda}-\lambda_{h}\right)+b_{h}\left(\lambda_{h}, P_{h} \hat{\lambda}-\lambda_{h}\right) \\
-a_{h}\left(u_{h} \mid u_{h}, \Pi_{h} \hat{u}-u_{h}\right)-b_{h}\left(\lambda_{h}, P_{h} \hat{\lambda}-\lambda_{h}\right) .
\end{aligned}
$$

The weak convergence (5.13) implies

$$
a\left(\hat{u} \mid \hat{u}, \hat{u}-u_{h}\right)+b\left(\hat{\lambda}, \hat{\lambda}-\lambda_{h}\right) \stackrel{h \rightarrow 0}{\longrightarrow} 0 .
$$

Using the boundedness of $\left\{\left(u_{h}, \lambda_{h}, \beta_{h}\right)\right\}_{h>0}$ and the approximation properties of $\Pi_{h}$ and $P_{h}$ due to Lemma 3.2 , we obtain

$$
a\left(u_{h} \mid u_{h}, \hat{u}-\Pi_{h} \hat{u}\right)+b\left(\lambda_{h}, \hat{\lambda}-P_{h} \hat{\lambda}\right) \stackrel{h \rightarrow 0}{\longrightarrow} 0 .
$$

The inequalities (3.12) and (3.13) imply

$$
\begin{aligned}
&-a\left(u_{h} \mid u_{h}, \Pi_{h} \hat{u}-u_{h}\right)+a_{h}\left(u_{h} \mid u_{h}, \Pi_{h} \hat{u}-u_{h}\right) \\
&-b\left(\lambda_{h}, P_{h} \hat{\lambda}-\lambda_{h}\right)+b_{h}\left(\lambda_{h}, P_{h} \hat{\lambda}-\lambda_{h}\right) \stackrel{h \rightarrow 0}{\longrightarrow} 0 .
\end{aligned}
$$

In order to prove that $\left\{\left(u_{h}, \lambda_{h}, \beta_{h}\right)\right\}_{h>0}$ converges strongly to $(\hat{u}, \hat{\lambda}, \hat{\beta})$, it suffices in view of (5.14)-(5.17) to show that

$$
a_{h}\left(u_{h} \mid u_{h}, \Pi_{h} \hat{u}-u_{h}\right)+b_{h}\left(\lambda_{h}, P_{h} \hat{\lambda}-\lambda_{h}\right) \stackrel{h \rightarrow 0}{\longrightarrow} 0
$$

holds.

In order to use the equality (4.3) it is necessary to show that $\Pi_{h} \hat{u}-u_{h} \in V_{h}^{0}$. Usually this will not be the case. But since $u_{h}$ and $\Pi_{h} \hat{u}$ are uniformly bounded in $W^{1, p}(\Omega)$, we modify the interpolant $\Pi_{h} \hat{u}$ into $\widehat{u}_{h}$ only along the slit $\Sigma$, such that

$$
\widehat{u}_{h}-u_{h} \in V_{h}^{0} \text {. }
$$

To this end we use a fixed sequence of finite element functions $\chi_{h} \in V_{h}$ with a jump of constant height 1 along $\Sigma$ satisfying $\left\|\chi_{h}\right\|_{W^{1,2}(\Omega)} \leq c$ and define

$$
\widehat{u}_{h}:=\Pi_{h} \widehat{u}+\left(\beta_{h}-\widehat{\beta}\right) \chi_{h} .
$$


Then still

$$
\left\|\widehat{u}_{h}-\widehat{u}\right\|_{W^{1,2}(\Omega)} \rightarrow 0 \text { for } h \rightarrow 0 .
$$

Now because of (5.19) the equalities (4.3), (4.4) with the test function $\widehat{u}_{h}-u_{h}$ and, together with the definitions (3.10), we obtain

$$
\begin{aligned}
& a_{h}\left(u_{h} \mid u_{h}, \widehat{u}_{h}-u_{h}\right)+b_{h}\left(\lambda_{h}, P_{h} \widehat{\lambda}-\lambda_{h}\right) \\
&=\quad \ell_{1}^{h}\left(\widehat{u}_{h}-u_{h}, \beta_{h}\right)+\left\langle\lambda_{h}, \widehat{u}_{h}-u_{h}\right\rangle \\
&+\ell_{2}^{h}\left(P_{h} \widehat{\lambda}-\lambda_{h}, \beta_{h}\right)-\left\langle u_{h}, P_{h} \widehat{\lambda}-\lambda_{h}\right\rangle+d_{h}\left(u_{h}, P_{h} \widehat{\lambda}-\lambda_{h}\right) \\
&+\int_{\Omega_{h}} \nabla \widetilde{\xi}_{h}\left(\widetilde{u}_{h}\right) \cdot \nabla\left(\widehat{u}_{h}-\widetilde{u}_{h}\right) d x \\
&(5.21)= \ell_{1}^{h}\left(\widehat{u}_{h}-u_{h}, \beta_{h}\right)-\ell_{1}\left(\widehat{u}_{h}-u_{h}, \widehat{\beta}\right) \\
&+\ell_{2}^{h}\left(P_{h} \widehat{\lambda}-\lambda_{h}, \beta_{h}\right)-\ell_{2}\left(P_{h} \widehat{\lambda}-\lambda_{h}, \widehat{\beta}\right) \\
&+d_{h}\left(u_{h}, P_{h} \widehat{\lambda}-\lambda_{h}\right)-d\left(u_{h}, P_{h} \widehat{\lambda}-\lambda_{h}\right) \\
&+\ell_{1}\left(\widehat{u}_{h}-\widehat{u}, \widehat{\beta}\right)+\ell_{1}\left(\widehat{u}-u_{h}, \widehat{\beta}\right)+\ell_{2}\left(P_{h} \widehat{\lambda}-\widehat{\lambda}, \widehat{\beta}\right)+\ell_{2}\left(\widehat{\lambda}-\lambda_{h}, \widehat{\beta}\right) \\
&+d\left(u_{h}, P_{h} \widehat{\lambda}-\widehat{\lambda}\right)+d\left(u_{h}, \widehat{\lambda}-\lambda_{h}\right) \\
&+\left\langle\lambda_{h}, \widehat{u}_{h}-\widehat{u}\right\rangle+\left\langle\lambda_{h}-\widehat{\lambda}, \widehat{u}\right\rangle \\
&-\left\langle u_{h}, P_{h} \widehat{\lambda}-\widehat{\lambda}\right\rangle-\left\langle u_{h}-\widehat{u}, \widehat{\lambda}\right\rangle \\
&+\int_{\Omega^{h}} \nabla \widetilde{\xi}\left(\widetilde{u}_{h}\right) \cdot \nabla\left(\widehat{u}_{h}-\widetilde{u}_{h}\right) d x .
\end{aligned}
$$

Using the result (5.12) and the boundedness of $\widehat{u}_{h}-\widetilde{u}_{h}$ in $V$, we have

$$
\int_{\Omega_{h}} \nabla \widetilde{\xi}_{h}\left(\widetilde{u}_{h}\right) \cdot \nabla\left(\widehat{u}_{h}-\widetilde{u}_{h}\right) d x \leq c\left\{\frac{1}{2} \int_{\Omega_{h}}\left|\nabla \xi_{h}\left(\widetilde{u}_{h}\right)\right|^{2} d x\right\}^{\frac{1}{2}}=c \sqrt{\mathcal{J}_{h}\left(\widetilde{u}_{h}\right)} \stackrel{h \rightarrow 0}{\longrightarrow} 0 .
$$

The continuity of the operator $\mathcal{K}$ in (1.29) implies the compactness of the bilinear form $d$ on $V$; hence the weak convergence (5.13) for the sequence $\left\{\lambda_{h}\right\}_{h>0}$ yields

$$
d\left(u_{h}, \widehat{\lambda}-\lambda_{h}\right)=\left\langle u_{h}, \mathcal{K}^{\prime}\left(\widehat{\lambda}-\lambda_{h}\right)\right\rangle \rightarrow 0 \text { for } h \rightarrow 0
$$

Thus (5.22), (5.23) imply together with (5.21), (3.14), (3.15), the weak convergence (5.13) and the approximation property of $\Pi_{h}$ and $P_{h}$ the result (5.19). Hence we have shown that

$$
\begin{aligned}
& u_{h} \rightarrow \widehat{u} \quad \text { strongly in } V, \\
& \lambda_{h} \rightarrow \widehat{\lambda} \quad \text { strongly in } H, \\
& \beta_{h} \rightarrow \widehat{\beta} \quad \text { in } \mathbb{R} \text { for } h \rightarrow 0 .
\end{aligned}
$$


4. Step: In the last step we show that $(\widehat{u}, \widehat{\lambda}, \widehat{\beta})$ must coincide with the solution $(u, \lambda, \beta)$ of (1.37). Using (4.3) for an arbitrary but fixed $v \in V$, we obtain the following inequality

$$
\begin{aligned}
\left|a(\widehat{u} \mid \widehat{u}, v)-\langle\widehat{\lambda}, v\rangle-\ell_{1}(v, \widehat{\beta})\right| & \\
\leq & \left\{\left|a(\widehat{u} \mid \widehat{u}, v)-a_{h}(\widehat{u} \mid \widehat{u}, v)\right|+\left|\left\langle\lambda_{h}-\widehat{\lambda}, v\right\rangle\right|\right. \\
& +\left|a_{h}(\widehat{u} \mid \widehat{u}, v)-a_{h}\left(u_{h} \mid u_{h}, v\right)\right|+\left|\ell_{1}^{h}\left(v, \beta_{h}\right)-\ell_{1}(v, \widehat{\beta})\right| \\
& \left.+\left|a_{h}\left(u_{h} \mid u_{h}, v\right)-\left\langle\lambda_{h}, v\right\rangle-\ell_{1}^{h}\left(v, \beta_{h}\right)\right|\right\} \\
\leq & \left\{\left|a(\widehat{u} \mid \widehat{u}, v)-a_{h}(\widehat{u} \mid \widehat{u}, v)\right|+\left|\left\langle\lambda_{h}-\widehat{\lambda}, v\right\rangle\right|\right. \\
& +\left|a_{h}(\widehat{u} \mid \widehat{u}, v)-a_{h}\left(u_{h} \mid u_{h}, v\right)\right|+\left|\ell_{1}^{h}\left(v, \beta_{h}\right)-\ell_{1}(v, \widehat{\beta})\right| \\
& +\left|a_{h}\left(u_{h} \mid u_{h}, v-R_{h} v\right)-\left\langle\lambda_{h}, v-R_{h} v\right\rangle-\ell_{1}^{h}\left(v-R_{h} v, \beta_{h}\right)\right| \\
& \left.+\left|\int_{\Omega_{h}} \nabla \widetilde{\xi}_{h}\left(\widetilde{u}_{h}\right) \cdot \nabla \widetilde{R}_{h} v d x\right|\right\} .
\end{aligned}
$$

Here $R_{h} v \in V_{h}$ is the Ritz projection of $v$ defined by (3.19). We obtain from (5.25), using Lemma 3.2, (3.12), (3.15), (5.11), (5.12) and (5.24),

$$
a(\widehat{u} \mid \widehat{u}, v)-\langle\widehat{\lambda}, v\rangle-\ell_{1}(v, \widehat{\beta})=0 \quad \text { for all } v \in V .
$$

In a similar way we obtain from (4.4) the inequality

$$
\begin{aligned}
\mid b(\widehat{\lambda}, \psi) & +\langle\widehat{u}, \psi\rangle-d(\widehat{u}, \psi)-\ell_{2}(\psi, \widehat{\beta}) \mid \\
\leq & \left\{\left|b\left(\widehat{\lambda}, \psi-P_{h} \psi\right)\right|+\left|b\left(\widehat{\lambda}-\lambda_{h}, P_{h} \psi\right)\right|+\left|\left\langle\widehat{u}-u_{h}, \psi\right\rangle\right|\right. \\
& +\left|b\left(\lambda_{h}, P_{h} \psi\right)-b_{h}\left(\lambda_{h}, P_{h} \psi\right)\right|+\left|\ell_{2}(\psi, \widehat{\beta})-\ell_{2}^{h}\left(\psi, \beta_{h}\right)\right| \\
& +\left|d\left(\widehat{u}-u_{h}, \psi\right)\right|+\left|d\left(u_{h}, \psi-P_{h} \psi\right)\right| \\
& +\left|d\left(u_{h}, P_{h} \psi\right)-d_{h}\left(u_{h}, P_{h} \psi\right)\right| \\
& \left.+\left|\ell_{2}^{h}\left(\psi-P_{h} \psi, \beta_{h}\right)\right|+\left|\left\langle u_{h}, \psi-P_{h} \psi\right\rangle\right|\right\} .
\end{aligned}
$$

Using the same arguments as for the proof of (5.26), we obtain the equality

$$
b(\widehat{\lambda}, \psi)+\langle\widehat{u}, \psi\rangle-d(\widehat{u}, \psi)-\ell_{2}(\psi, \widehat{\beta})=0 \quad \text { for all } \psi \in H .
$$

Since $\widehat{u} \in V \cap K_{\widetilde{s}_{0}}$ defines a subsonic flow, Theorem 1.3 implies the validity of the Kutta-Joukowski condition at $T E$.

Thus we have proven that $(\widehat{u}, \hat{\lambda}, \widehat{\beta})$ is a subsonic flow solution of (1.37). The assumption on the uniqueness of such a solution implies that $(\widehat{u}, \widehat{\lambda}, \widehat{\beta})=(u, \lambda, \beta)$ holds and moreover, that the whole sequence of solutions $\left\{\left(u_{h}, \lambda_{h}, \beta_{h}\right)\right\}_{h>0}$ of the minimization problem (4.5) converges to the solution. 
Note that due to the uniform $L^{\infty}$-bound for the gradients of the sequence $\left\{u_{h}\right\}_{h>0}$, the latter converges in the $W^{1, p}(\Omega)$-norms for $p \in[1, \infty)$.

5.2. The case of transonic flow. The purpose of this section is a convergence proof for the sequence $\left\{\left(\widetilde{u}_{h}, \widetilde{\lambda}_{h}, \beta_{h}\right)\right\}_{h>0}$ of solutions of problem (4.5). The main underlying ideas for this proof go back to Berger [6], who has proved the convergence of the interior problem with a simplified boundary condition on polygonal domains. An extension of this work to domains with arbitrary curved boundaries can be found in Berger and Feistauer [7]. Therefore we shall now state the corresponding main results, which enable us to show the convergence of the exterior coupled problem.

Lemma 5.3. Let $\left\{\mathcal{T}_{h}\right\}_{h>0}$ be a family of triangulations satisfying the uniform angle property. This implies that the supports of the hat functions $w_{h_{i}}$ contain at most a certain finite number of triangles independent of $h$. This in turn implies that there is a constant $c>0$ such that

$$
A_{i} \leq c h^{2} \text { for } i=1, \ldots, N \text { and all } 0<h \leq 1
$$

Then a family of conforming solutions $u_{h}$ to (4.5), resp. (3.11), satisfies the estimate

$$
-\int_{\Omega} \nabla u_{h} \cdot \nabla v_{h} d x \leq B \int_{\Omega} v_{h} d x+c h^{\varepsilon-1}\left\|v_{h}\right\|_{L^{\infty}(\Omega)} \text { for all } v_{h} \in E_{h}
$$

with $2>\varepsilon>1$ and $B$ as in (4.2) where

$$
E_{h}:=\left\{v_{h} \in V_{h} \mid v_{h} \geq 0 \text { and } v_{h}=0 \text { on } \overline{\Gamma_{\infty} \cup \Sigma}\right\} .
$$

Remark. Note that this result is slightly stronger than Berger's in [6, Theorem 4.1].

Proof. The nonnegativity of $v_{h} \in E_{h}$ implies $\widetilde{v}_{h}=\sum_{i=1}^{N} \alpha_{i} w_{h_{i}}$ with $\alpha_{j} \geq 0$ according to the correspondence between $v_{h}$ and $\widetilde{v}_{h}$ in Section 3. By using $\left\|\widetilde{v}_{h}\right\|_{\infty}=$ $\max _{i=1, \ldots, N} \alpha_{i}$, this gives

$$
\begin{aligned}
& -\int_{\Omega_{h}} \nabla \widetilde{u}_{h} \cdot \nabla \widetilde{v}_{h} d x-B \int_{\Omega_{h}} \widetilde{v}_{h} d x \\
= & \sum_{\substack{i=1 \\
p_{i} \notin \Sigma \cup \Gamma_{\infty}}}^{N} \alpha_{i}\left(-\int_{\Omega_{h}} \nabla \widetilde{u}_{h} \cdot \nabla w_{h_{i}} d x-B \int_{\Omega_{h}} w_{h_{i}} d x\right) \\
\leq & \left\|\widetilde{v}_{h}\right\|_{\infty} \sum_{\substack{i=1 \\
p_{i} \notin \Sigma \cup \Gamma_{\infty}}}^{N}\left[-\int_{\Omega_{h}} \nabla \widetilde{u}_{h} \cdot \nabla w_{h_{i}} d x-B \int_{\Omega_{h}} w_{h_{i}} d x\right]^{+} .
\end{aligned}
$$


The Cauchy-Schwarz inequality gives with (4.2)

$$
\begin{aligned}
& \sum_{\substack{i=1 \\
p_{i} \notin \Sigma \cup \Gamma_{\infty}}}^{N}\left[-\int_{\Omega_{h}} \nabla \widetilde{u}_{h} \cdot \nabla w_{h_{i}} d x-B \int_{\Omega_{h}} w_{h_{i}} d x\right]^{+} \\
& \leq\left(\sum_{i=1}^{N} A_{i}^{\varepsilon}\right)^{\frac{1}{2}}\left\{\sum_{\substack{i=1 \\
p_{i} \notin \Sigma \cup \Gamma_{\infty}}}^{N} \frac{1}{A_{i}^{\varepsilon}}\left(\left[-\int_{\Omega_{h}} \nabla \widetilde{u}_{h} \cdot \nabla w_{h_{i}} d x-B \int_{\Omega_{h}} w_{h_{i}} d x\right]^{+}\right)^{2}\right\}^{\frac{1}{2}} \\
& \quad=\left(\sum_{i=1}^{N} A_{i}^{\varepsilon}\right)^{\frac{1}{2}} \sqrt{\frac{2}{\mu} \mathcal{P}_{h}\left(\widetilde{u}_{h}\right)} \\
& \leq\left(\max _{i=1, \ldots, N} A_{i}^{\varepsilon-1}\right)^{\frac{1}{2}}\left(\sum_{i=1}^{N} A_{i}\right)^{\frac{1}{2}} \sqrt{\frac{2}{\mu} \mathcal{P}_{h}\left(\widetilde{u}_{h}\right)}
\end{aligned}
$$

By our assumptions on the mesh we have

$$
A_{i} \leq C h^{2} \quad \text { and } \quad \sum_{i=1}^{N} A_{i} \leq C \int_{\Omega_{h}} d x=C\left|\Omega_{h}\right|
$$

By (4.5) we have the uniform bound

$$
0 \leq \mathcal{J}_{h}\left(\widetilde{u}_{h}\right) \leq \mathcal{J}_{h}(0)=\frac{1}{2}\left\|\nabla \xi_{h}(0)\right\|^{2}+\mathcal{P}_{h}(0) .
$$

Therefore (5.30) implies with the above estimates the inequality

$$
-\int_{\Omega_{h}} \nabla \widetilde{u}_{h} \cdot \nabla \widetilde{v}_{h} d x-B \int_{\Omega_{h}} \widetilde{v}_{h} d x \leq c h^{\varepsilon-1}\left\|\widetilde{v}_{h}\right\|_{\infty}=c h^{\varepsilon-1}\left\|v_{h}\right\|_{\infty},
$$

since $\left\|\widetilde{v}_{h}\right\|_{\infty}=\left\|v_{h}\right\|_{\infty}$. Further, we have

$$
\left|\int_{\Omega_{h}} \widetilde{v}_{h} d x-\int_{\Omega} v_{h} d x\right| \leq\left|\int_{\Omega \backslash \Omega_{h}} v_{h} d x\right|+\left|\int_{\Omega_{h} \backslash \Omega} \widetilde{v}_{h} d x\right| \leq \operatorname{ch}^{2}\left\|v_{h}\right\|_{\infty}
$$

due to [32]. Correspondingly, there holds

$$
\begin{aligned}
& \left|\int_{\Omega} \nabla u_{h} \cdot \nabla v_{h} d x-\int_{\Omega_{h}} \nabla \widetilde{u}_{h} \cdot \nabla \widetilde{v}_{h} d x\right| \\
& \quad \leq\left|\int_{\Omega \backslash \Omega_{h}} \nabla u_{h} \cdot \nabla v_{h} d x\right|+\left|\int_{\Omega_{h} \backslash \Omega} \nabla \widetilde{u}_{h} \cdot \nabla \widetilde{v}_{h} d x\right| \\
& \quad \leq \widetilde{s}_{0}\left(\left\|\nabla v_{h}\right\|_{L_{\infty}\left(\Omega \backslash \Omega_{h}\right)}+\left\|\nabla \widetilde{v}_{h}\right\|_{L_{\infty}\left(\Omega_{h} \backslash \Omega\right)}\right)\left(\left|\Omega \backslash \Omega_{h}\right|+\left|\Omega_{h} \backslash \Omega\right|\right) \\
& \leq \widetilde{s}_{0} c\left\|v_{h}\right\|_{\infty} h
\end{aligned}
$$

due to the inverse estimate [18, Theorem 3.2.6]. Since $1<\varepsilon<2$, we have $h \leq h^{1-\varepsilon}$ for $0<h \leq 1$ and (5.29) follows. 
Lemma 5.4. Under the assumptions of Lemma 5.3 let $\left\{\widetilde{u}_{h}\right\}_{h>0}$ be a sequence of solutions to problem (4.5) with $h \rightarrow 0$ and let $\left\{u_{h}\right\}_{h>0}$ be the corresponding sequence of conforming approximations defined in Section 3. Then there exists a subsequence $\left\{\widetilde{u}_{h}\right\}_{h>0}$ and a function $u \in V \cap K_{s_{0}}$ such that the strong convergence

$$
\left\|u-u_{h}\right\|_{W^{1, p}(\Omega)} \stackrel{h \rightarrow 0}{\longrightarrow} 0 \text { for all } p \in[1, \infty)
$$

and the entropy inequality

$$
-\int_{\Omega} \nabla u \cdot \nabla v d x \leq B^{\prime} \int_{\Omega} v d x \quad \text { for all } v \in E
$$

hold for an appropriate $B^{\prime}>0$ where

$$
E:=\left\{v \in W^{1,2}(\Omega) \mid v \geq 0 \text { and } v=0 \text { on } \overline{\Gamma_{\infty} \cup \Sigma}\right\} .
$$

Proof. The sequence $\left\{u_{h}\right\}_{h>0}$ satisfies (5.29). The Sobolev embedding theorem applied to the case $W^{1, q}(\Omega) \subset L^{\infty}(\Omega)$ for $q>2$ gives for all $v_{h} \in E_{h}$

$$
\mathcal{G}_{h}^{B}\left(v_{h}\right):=\int_{\Omega} \nabla u_{h} \cdot \nabla v_{h} d x+B \int_{\Omega} v_{h} d x \geq-c h^{\varepsilon-1}\left\|v_{h}\right\|_{1, q} .
$$

Choosing $B^{\prime}$ large enough there exists an $h_{0}>0$ such that for any $h$ with $0<h<h_{0}$ we obtain for $B^{\prime}>c h_{0}^{\varepsilon-1}+B$ the estimate

$$
\mathcal{G}_{h}^{B^{\prime}}\left(v_{h}\right) \geq 0 .
$$

Due to $\left\|\nabla u_{h}\right\| \leq|\Omega|^{\frac{1}{2}}\left\|\nabla u_{h}\right\|_{\infty} \leq|\Omega|^{\frac{1}{2}} s_{0}$ we have with an appropriate constant $c>0$

$$
\left|\mathcal{G}_{h}^{B^{\prime}}\left(v_{h}\right)\right| \leq\left\|\nabla u_{h}\right\| \cdot\left\|\nabla v_{h}\right\|+B|\Omega|^{\frac{1}{2}}\left\|v_{h}\right\| \leq c\left\|v_{h}\right\|_{1,2} .
$$

This implies $\mathcal{G}_{h}^{B^{\prime}} \in\left[W^{1,2}(\Omega)\right]^{\prime}$ and thereby $\mathcal{G}_{h}^{B^{\prime}} \in\left[W^{1, q}(\Omega)\right]^{\prime}$ for $q \geq 2$ since $\Omega$ is a bounded domain. The sequence is bounded and therefore has a weakly convergent subsequence. The Corollary to Lemma 3.1 in Mandel and Nečas [44] gives $\mathcal{G}_{h}^{B^{\prime}} \rightarrow \mathcal{G}$ in $\left[W^{1, q}(\Omega)\right]^{\prime}$ for $q>2$. As in the first part of the proof of Feistauer and Nečas [31, Theorem 4.23] this implies the strong convergence $u_{h} \rightarrow u$ in $W^{1,2}(\Omega)$. Since $\left\|\nabla u_{h}\right\|_{\infty}$ is bounded by $s_{0}$ this implies convergence in $W^{1, p}(\Omega)$ for $p \in[1, \infty)$.

The inequality $\mathcal{G}_{h}^{B^{\prime}}\left(v_{h}\right) \geq 0$ implies (5.32) in the limit when taking $v_{h} \rightarrow v$ in $W^{1,2}(\Omega)$.

For the following convergence proof we have to modify and extend the density $\rho$ in order to obtain a function $\widetilde{\rho}:[0, \infty) \rightarrow[0, \infty)$ such that $\widetilde{\rho}(s) \geq \rho_{c}$ for some constant $\rho_{c}>0$. We choose $s^{*} \in\left(s_{0}, \frac{2 a_{0}^{2}}{\kappa-1}\right)$, set $\widetilde{\rho}(s)=\rho(s)$ for $s \in\left[0, s^{*}\right]$ and use the extension described in Feistauer and Nečas [31] whereby $\widetilde{\rho}(s)=\rho_{c}$ for large $s$. Note that the set of solutions to problem (1.37) is not changed by this modification.

Theorem 5.5. Suppose that the following assumptions hold:

(a) Let the family of triangulations be quasiuniform. 
(b) The problem (1.37) has exactly one solution $(u, \lambda, \beta) \in\left(V \cap K_{s_{0}}\right) \times H \times I$, which satisfies the entropy condition (5.32).

(c) The solution of (1.37) satisfies the subsonic flow condition (1.38) near the trailing edge.

Then there exists a constant $\widetilde{s}_{0} \geq s_{0}$ such that a sequence of solutions $\left\{\left(\widetilde{u}_{h}, \widetilde{\lambda}_{h}, \beta_{h}\right)\right\}_{h>0}$ of the modified minimization problem

$$
\mathcal{J}_{h}\left(\widetilde{u}_{h}\right)=\min _{\tilde{\varphi}_{h} \in \widetilde{V}_{h} \cap K_{\tilde{s}_{0}}} \mathcal{J}_{h}\left(\widetilde{\varphi}_{h}\right)
$$

subject to (4.3), (4.4) and $F\left(\beta_{h}\right)=0$ converges to the unique solution $(u, \lambda, \beta)$ of the variational problem (1.37).

Proof. We shall split the proof into three steps.

Step 1: The assumption (3.20) implies that $\widetilde{R}_{h} u \in \widetilde{V}_{h} \cap K_{c^{*} \cdot s_{0}}$. We modify $\widetilde{R}_{h} u$ along the slit such that the modified function $\widetilde{u}_{h}^{I}$ satisfies the Kutta-Joukowski condition. This modification is the same as the one described in the proof of Lemma 5.1 and in [10] Since $\widetilde{R}_{h} u$ is uniformly bounded in $W^{1, \infty}\left(\Omega_{h}\right)$, the modified function will also be uniformly bounded in $W^{1, \infty}\left(\Omega_{h}\right)$. Therefore, we can find a constant $\widetilde{s}_{0} \geq c^{*} \cdot s_{0}$, such that

$$
\left\|\nabla \widetilde{u}_{h}^{I}\right\|_{\infty} \leq \widetilde{s}_{0}
$$

holds. Moreover, the modified function has the same approximation properties as $\widetilde{R}_{h} u$, which implies

$$
\left\|u-u_{h}^{I}\right\|_{W^{1,2}(\Omega)} \rightarrow 0 \text { for } h \rightarrow 0 .
$$

Step 2: Since the density $\rho$ is now modified, the functional $\mathcal{J}_{h}$ may be applied to $\widetilde{u}_{h}^{I} \in \widetilde{V}_{h} \cap K_{\widetilde{s}_{0}}$; and we obtain from (5.34) the inequality

$$
0 \leq \mathcal{J}_{h}\left(\widetilde{u}_{h}\right) \leq \mathcal{J}_{h}\left(\widetilde{u}_{h}^{I}\right)=\frac{1}{2} \int_{\Omega_{h}}\left|\nabla \widetilde{\xi}_{h}\left(\widetilde{u}_{h}^{I}\right)\right|^{2} d x+\mathcal{P}_{h}\left(\widetilde{u}_{h}^{I}\right) .
$$

By using the same arguments as in the proof of (5.12) we get with the help of (5.35)

$$
\frac{1}{2} \int_{\Omega_{h}}\left|\nabla \widetilde{\xi}_{h}\left(\widetilde{u}_{h}^{I}\right)\right|^{2} d x \rightarrow 0 \text { for } h \rightarrow 0 .
$$

Note that our function $\widetilde{u}_{h}^{I}$ coincides with $\widetilde{R}_{h} u$ in the points $p_{i}$ for $i=2 L+1, \ldots, N$. Since our penalty functional has no contributions from nodes belonging to $\Sigma$ we have $\mathcal{P}_{h}\left(\widetilde{u}_{h}^{I}\right)=\mathcal{P}_{h}\left(\widetilde{R}_{h} u\right)$. Further, we have by the definition of the Ritz projection (3.19), by the fact that $u$ satisfies the entropy inequality (5.32), and by the nonnegativity 
of $\widetilde{w}_{h_{i}}$, that

$$
\begin{aligned}
0 \leq & \mathcal{P}_{h}\left(\widetilde{R}_{h} u\right) \\
= & \frac{\mu}{2} \sum_{\substack{i=1 \\
p_{i} \notin \Sigma \cup \Gamma_{\infty}}}^{N} \frac{1}{\left(A_{i}\right)^{\varepsilon}}\left|\left[-\int_{\Omega_{h}} \nabla \widetilde{R}_{h} u \cdot \nabla \widetilde{w}_{h_{i}} d x-B \int_{\Omega_{h}} \widetilde{w}_{h_{i}} d x\right]^{+}\right|^{2} \\
\leq & \frac{\mu}{2} \sum_{\substack{i=1 \\
p_{i} \notin \Sigma \cup \Gamma_{\infty}}}^{N} \frac{1}{\left(A_{i}\right)^{\varepsilon}}\left|\left[-\int_{\Omega} \nabla u \cdot \nabla w_{h_{i}} d x-B \int_{\Omega} w_{h_{i}} d x\right]^{+}\right|^{2} \\
= & \left.\frac{\mu B}{2} \sum_{\substack{i=1 \\
p_{i} \notin \Sigma \cup \Gamma_{\infty}}} \frac{1}{\left(A^{i}\right)^{\varepsilon}}\left|\left[\int_{\Omega} w_{h_{i}} d x-\int_{\Omega_{h}} \widetilde{w}_{h_{i}} d x\right]^{+}\right|^{2} \sum_{\substack{i=1 \\
p_{i} \notin \Sigma \cup \Gamma_{\infty}}}^{N} \frac{1}{\left(A^{i}\right)^{\varepsilon}}\left[\int_{\Omega \backslash \Omega_{h}} w_{h_{i}} d x\right]^{2}\right]_{\substack{i=1 \\
p_{i} \notin \Sigma \cup \Gamma_{\infty}}}^{N} \frac{1}{A_{i}^{\varepsilon}}\left\{\left(\text { meas supp } w_{h_{i}}\right) \cap \omega_{P}^{h}\right\}^{2} . \\
\leq & \frac{\mu B}{2} \sum^{N} .
\end{aligned}
$$

Since the grid satisfies the uniform angle property and $P$ is piecewise smooth we have meas $\left(\operatorname{supp} w_{h_{i}}\right) \cap \omega_{P}^{h} \leq c h_{i}^{3}$ and $c h_{i}^{2} \leq A_{i}$. Hence, we obtain

$$
0 \leq \mathcal{P}_{h}\left(\widetilde{R}_{h} u\right) \leq c \sum_{\substack{i=1 \\ p_{i} \notin \Sigma \cup \Gamma_{\infty}}}^{N} h_{i}^{2} h_{i}^{4-2 \varepsilon} \leq c^{\prime} h^{2(2-\varepsilon)} .
$$

If we combine this with (5.36) and (5.37) we obtain the result

$$
\mathcal{J}_{h}\left(\widetilde{u}_{h}\right) \rightarrow 0 \text { for } h \rightarrow 0 \text {. }
$$

Step 3: We now apply Lemmata 5.3 and 5.4. We get the existence of a subsequence $\left\{\widetilde{u}_{h}\right\}_{h>0}$ and a function $\widehat{u} \in K_{\widetilde{s}_{0}}$ such that

$$
\begin{aligned}
\left\|\widehat{u}-u_{h}\right\|_{W^{1, p}(\Omega)} \stackrel{h \rightarrow 0}{\longrightarrow} 0, & \text { for all } p \in[1, \infty), \\
-\int_{\Omega} \nabla \widehat{u} \cdot \nabla v d x \leq B \int_{\Omega} v d x & \text { for all } v \in E .
\end{aligned}
$$

The convergent subsequence $\left\{u_{h}\right\}_{h>0}$ is bounded. Then (3.11) holds. Taking $\psi_{h}=$ $\lambda_{h}$ and using the coercivity (1.34) gives the boundedness of the corresponding sequence $\left\{\lambda_{h}\right\}_{h>0}$ in $H$. We may derive the analogue of (5.9) for $\widehat{\lambda}-\lambda_{h}$. Then the strong convergence (5.39) together with the boundedness of $\left\{\lambda_{h}\right\}_{h>0}$ in $H$ implies

$$
\begin{gathered}
\left\|\widehat{\lambda}-\lambda_{h}\right\|_{H^{-\frac{1}{2}}\left(\Gamma_{\infty}\right)} \stackrel{h \rightarrow 0}{\longrightarrow} 0, \\
b(\widehat{\lambda}, \psi)+\langle\widehat{u}, \psi\rangle-d(\widehat{u}, \psi)=\ell_{2}(\psi, \widehat{\beta}) \text { for all } \psi \in H .
\end{gathered}
$$

Using the same arguments as in Step 4 of Theorem 5.2 together with (5.39)-(5.41) and assumptions (a), (b) we obtain the result. 
Remark. The result of Theorem 5.5 is a generalization of the results proved in the papers by Berger in [6] and [5].

\section{NumERICAL RESUlts}

We present some results of our numerical computations made in 1989. Here, we compare three different treatments of the farfield boundary condition.

The first condition corresponds to the condition (1.8) which is just the parallel flow at infinity.

In the second case we first compute the FEM-BEM approximation to the harmonic solution $U$ of the exterior incompressible flow problem,

$$
\begin{aligned}
& \Delta U=0 \quad \text { in } \Omega \cup \Omega^{c}, \\
& \partial_{n} U=0 \text { on } \Gamma_{p} \text { and } \nabla U \rightarrow \vec{v}_{\infty} \text { at infinity , } \\
& U^{+}-U^{-}=\widetilde{\beta} \text { and } \partial_{n} U^{+}-\partial_{n} U^{-}=0 \text { on } \Sigma \text { subject to } F(\widetilde{\beta})=0 \text {. }
\end{aligned}
$$

Note, that our algorithm for the full coupling procedure needs only a slight modification to obtain $U$. With $U$, we used as a second boundary condition

$$
\rho\left(|\nabla u|^{2}\right) \partial_{n} u=\rho\left(\left|\vec{v}_{\infty}\right|^{2}\right) \partial_{n} U \text { on } \Gamma_{\infty} .
$$

The third case shows the results of the complete coupling described in Section 4.

For better comparison we give results for two standard test cases of flows around the NACA-0012 profile. Two different sized C-grids were used, see Figure 3. Here $\Gamma_{\infty}$ has two corner points where the $\mathcal{C}^{\infty}$ assumption used in the foregoing analysis is violated; however, due to the remark in Section 1.2, our convergence result can be extended to this simple Lipschitz curve. A large computational domain with 115 by 15 nodes, outer boundary 6 chord lengths from the profile; and a smaller

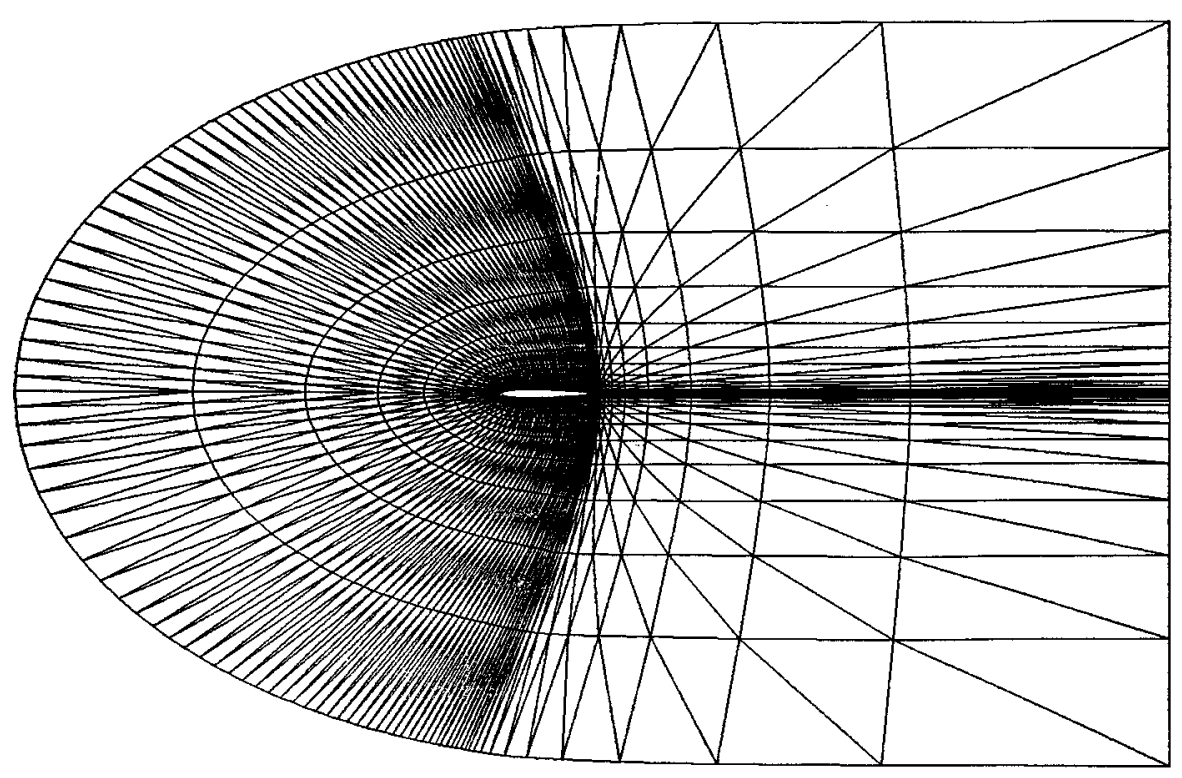

FiguRE 3. Large computational domain, containing the small subdomain 
subdomain with 111 by 13 nodes, outer boundary 3 chord lengths from the profile. We give the lift coefficients $c_{a}$ calculated from the pressure distribution along the profile.

Test Case 1: (purely subsonic flow)

\begin{tabular}{|l|c|c|}
\multicolumn{3}{|c}{$M_{\infty}=0.63 \quad \alpha=2 .{ }^{0}$} \\
\hline & $c_{a}$ large domain & $c_{a}$ small domain \\
\hline 1st case & 0.3470 & 0.3684 \\
\hline 2nd case & 0.3395 & 0.3481 \\
\hline full coupling & 0.3371 & 0.3391 \\
\hline
\end{tabular}

In a research report Kroll and Jain [41] give a lift coefficient of $c_{a}=0.3333$. They used an $O$-grid with 256 by 64 nodes, outer boundary 50 chord lengths from the profile. Their calculation was done with a potential and with an Euler code. Test Case 2: (transonic flow)

$$
M_{\infty}=0.8 \quad \alpha=1.25^{0}
$$

\begin{tabular}{|l|c|c|}
\hline & $c_{a}$ large domain & $c_{a}$ small domain \\
\hline 1st case & 0.4853 & 0.5852 \\
\hline 2nd case & 0.4705 & 0.5257 \\
\hline full coupling & 0.4209 & 0.4459 \\
\hline
\end{tabular}

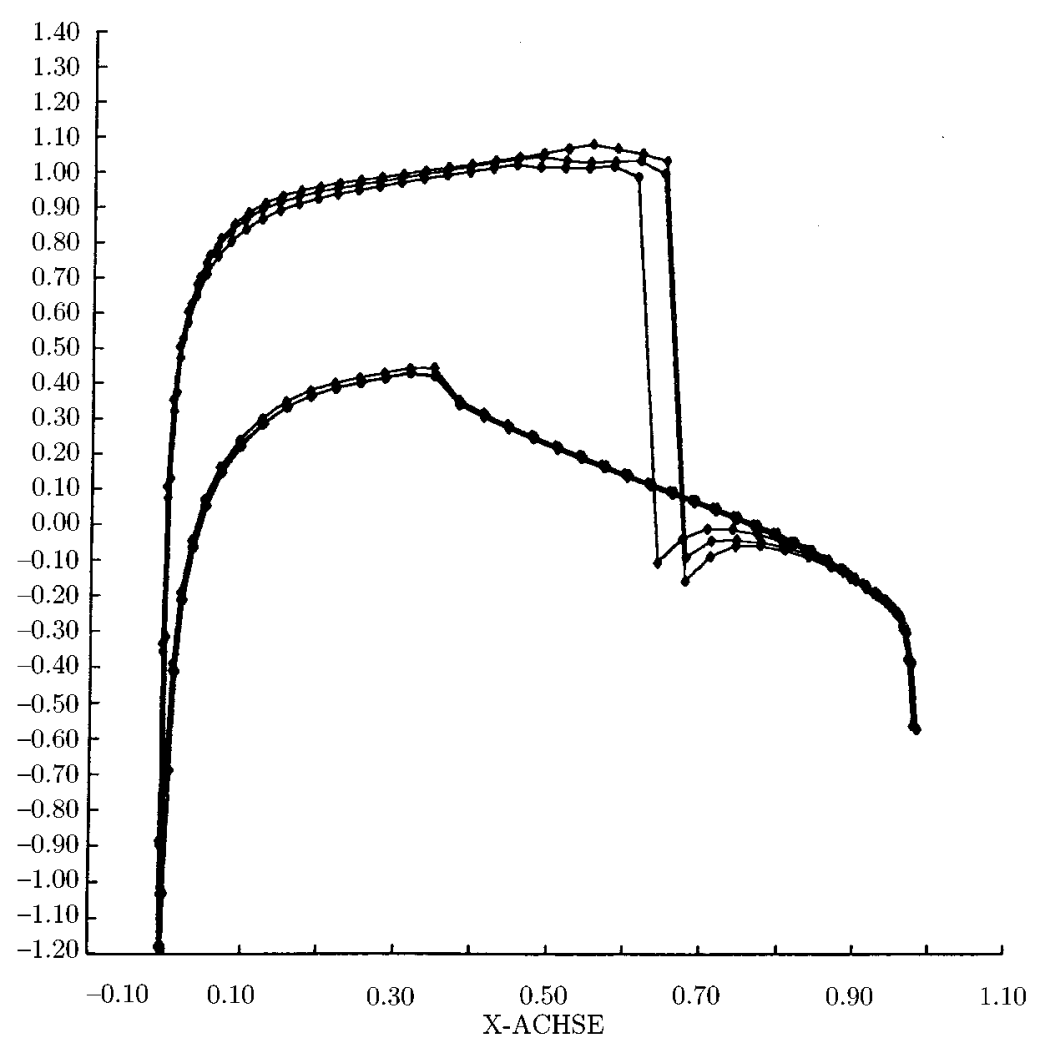

Figure 4. Pressure distributions for the transonic test case 2 on the large domain 


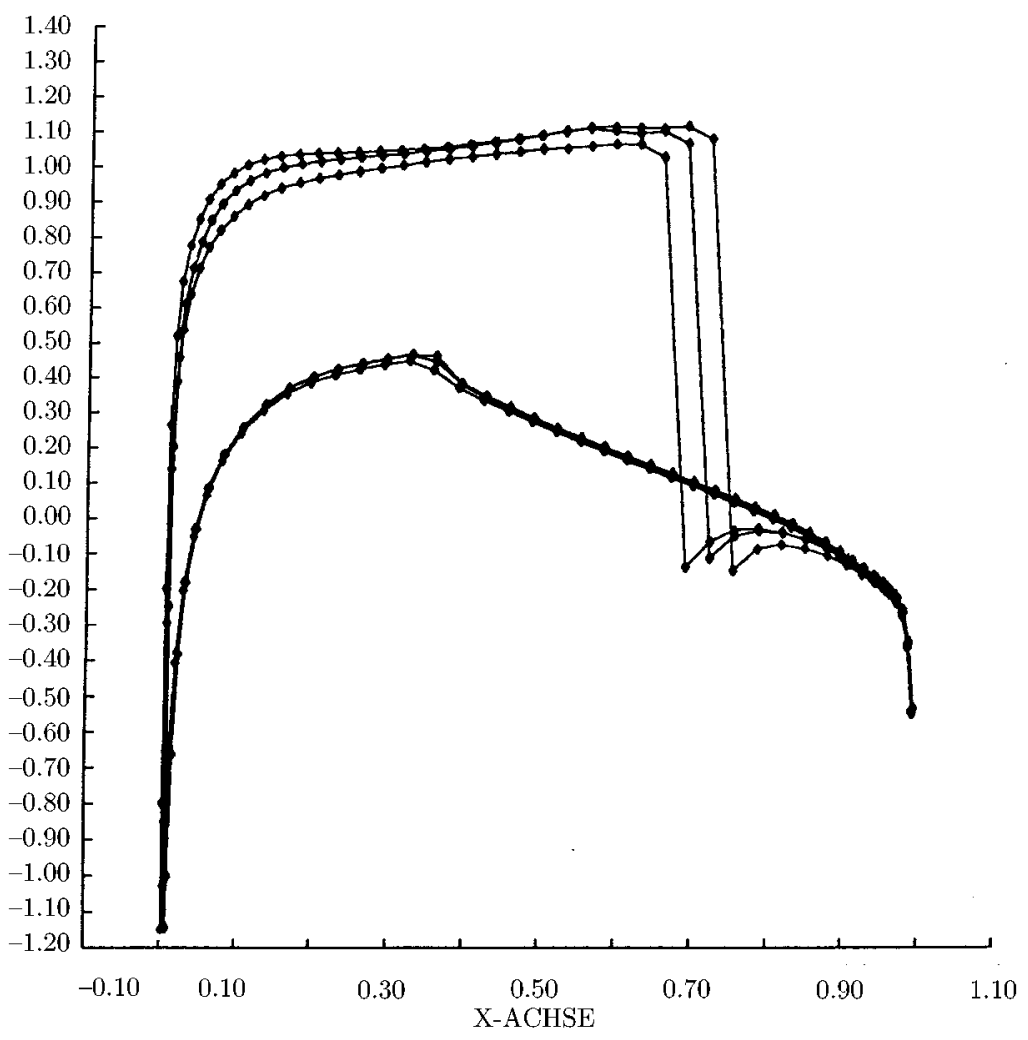

FiguRE 5. Pressure distributions for the transonic test case 2 on the small domain

In Rizzi and Viviant [53] a number of solutions for this test case is given. These were obtained by finite differences and finite volume discretizations of the full potential equation. The calculated lift coefficients vary between 0.5 and 1.1. In the AGARD Report Nr. 211 [1] newer results for the Euler equations were published. There the lift coefficients vary between 0.35 and 0.37 .

The improvement in the lift coefficients corresponds to a movement of the shock location to an upstream position and a slight reduction in shock strength. This is shown in Figures 4 and 5 for the above cases.

\section{ACKNOWLEDGEMENT}

The authors would like to thank the referee for his critical and helpful remarks.

\section{REFERENCES}

[1] AGARD Advisory Report No. 211, Test Cases for Inviscid Flow Field Methods, Report of Fluid Dynamics Panel Working Group 07, 1985.

[2] D. N. Arnold and W.L. Wendland, "The convergence of spline collocation for strongly elliptic equations on curves", Numer. Math. 47, 317-341 (1985). MR 87f:65142

[3] I. Babuška and A. K. Aziz, "Survey lectures on the mathematical foundations of the finite element method", in The Mathematical Foundation of the Finite Element Method with Applications to Partial Differential Equations, A.K. Aziz (ed.), Academic Press, New York 1-359 (1972). MR 54:9111 
[4] E. B. Becker, G. F. Carey and J. T. Oden, Finite Elements, Vol. VI: Fluid Mechanics, Prentice-Hall Inc., Englewood Cliffs, New Jersey, 1984.

[5] H. Berger, "Finite-Element-Approximationen für transsonische Strömungen", Doctoral Thesis, Universität Stuttgart, Germany, 1989.

[6] H. Berger, "A convergent finite element formulation for transonic flows", Numer. Math. 56, 425-447 (1989). MR 91g:65204

[7] H. Berger and M. Feistauer, "Analysis of the finite element variational crimes in the numerical approximation of transonic flow". Math. Comp. 61, 493-521 (1993). MR 94a:65055

[8] H. Berger, G. Warnecke and W. Wendland, "Finite Element-Berechnungen für transsonische Strömungen unter Berücksichtigung verschiedener Fernfeldrandbedingungen." In: Strömungen mit Ablösungen, DGLR-Bericht 88-05, Bonn (1988) 233-242.

[9] H. Berger, G. Warnecke and W. Wendland, "Finite elements for transonic potential flows," Numer. Meth. Part. Diff. Eqns. 6, 17-42 (1990). MR 91h:76053

[10] H. Berger, G. Warnecke and W.L. Wendland, "Coupling of FEM and BEM for transonic flows", in The Mathematics of Finite Elements and Applications (J.R. Whiteman ed.) John Wiley \& Sons, Chichester, 323-350 (1994). MR 95f:76053

[11] L. Bers, "Mathematical aspects of subsonic and transonic gas dynamics," in Surveys in Applied Mathematics III, Wiley, New York, 1958. MR 20:2960

[12] L. Bers, "Existence and uniqueness of subsonic flow past a given profile," Commun. Pure Appl. Math. 7, 441-504 (1954). MR 16:417a

[13] B. Bojarski, "Subsonic flow of compressible fluid," in Mathematical Problems in Fluid Mechanics, Polish Academy of Sciences, Warsaw, 1967. MR 37:4995

[14] H. Brezis and G. Stampacchia, "Une nouvelle méthode pour l'étude d'écoulements stationnaires," C. R. Acad. Sci. Paris 276, 129-132 (1973). MR 47:4521

[15] M. O. Bristeau, R. Glowinski, J. Periaux, P. Perrier, O. Pironneau and G. Poirier, "Application of optimal control and finite element methods to the calculation of transonic flows and incompressible flows," in Numerical Methods in Applied Fluid Dynamics, B. Hunt (ed.), Academic Press, New York, 203-312 (1980). MR 83h:65120

[16] M. O. Bristeau, R. Glowinski, J. Periaux, P. Perrier, O. Pironneau and G. Poirier, "Transonic flow simulations by finite elements and least squares methods," in Finite Elements in Fluids IV, R. H. Gallagher, G. Carey, J. T. Oden, and O. C. Zienkiewicz (eds.), Wiley, Chichester, 453-482 (1982).

[17] M. O. Bristeau, R. Glowinski, J. Periaux, P. Perrier, O. Pironneau and G. Poirier, "On the numerical solution of nonlinear problems in fluid dynamics by least squares and finite element methods II. Application to transonic flow simulations," Comput. Methods Appl. Mech. Engrg. 51, 363-394 (1985). MR 87d:76100

[18] P. G. Ciarlet, The Finite Element Method for Elliptic Problems, North-Holland, Amsterdam, 1978. MR 58:25001

[19] J. F. Ciavaldini, M. Pogu and G. Tournemine, "Une nouvelle approche dans le plain physique pour le calcul d'écoulements subcritiques et stationaires autor d'un profil portant," $J$. de Mécanique 16, 257-288 (1977). MR 56:10418

[20] J. F. Ciavaldini, M. Pogu and G. Tournemine, "Existence and regularity of stream functions for subsonic flows past profiles with a sharp trailing edge," Arch. Rat. Mech. Anal. 93, 1-14 (1986). MR 87d:76108

[21] C. Coclici and W.L. Wendland, "On the treatment of the Kutta-Joukowski condition in transonic flow computations", in preparation (Preprint 95-14 MIA Univ. Stuttgart).

[22] M. Costabel, "Boundary integral operators on Lipschitz domains: Elementary results," SIAM J. Math. Anal. 19, 613-626 (1988). MR 89h:35090

[23] R. Courant and D. Hilbert, Methods of Mathematical Physics II, Interscience Publishers, New York, 1962. MR 25:4216

[24] R. Courant and K. O. Friedrichs, Supersonic Flow and Shock Waves, Wiley, New York (1948). [Reprinted by Springer-Verlag, New York (1985).] MR 10:637c; MR 54:9284

[25] M. Crouzeix and V. Thomée, "The stability on $L_{p}$ and $W_{p}^{1}$ of the $L_{2}$-projection onto finite element function spaces", Math. Comp. 48, 521-532 (1987). MR 88f:41016

[26] M. Dauge and M. Pogu, "Existence et régularité de la function potentiel pour des écoulements subcritiques s'établissant autour d'un corps à singularité conique," Ann. Fac. des Sci. de Toulouse IX, 213-242 (1988). CMP 97:05 
[27] M. Djaoua, "A method of calculation of lifting flows around two-dimensional corner-shaped bodies," Math. Comp. 36, 405-425 (1981). MR 82c:76011

[28] H. Federer, Geometric Measure Theory, Springer-Verlag, Berlin-Heidelberg-New York (1969). MR 41:1976

[29] M. Feistauer, J. Felcman, M. Rokyta and Z. Vlášek, "Finite-element solution of flow problems with trailing conditions," J. Comp. Appl. Math. 44, 131-165 (1992). MR 94d:76052

[30] M. Feistauer, G.C. Hsiao, R.E. Kleinman and R. Tezaur, "Analysis and numerical realization of coupled BEM and FEM for nonlinear exterior problems", in Inverse Scattering and Potential Problems in Mathematical Physics (R.E Kleinman, R. Kress and E. Martensen eds.), Verlag Peter Lang, Frankfurt, 47-73 (1995). MR 95m:76045

[31] M. Feistauer and J. Nečas, "On the solvability of transonic potential flow problems," $Z$. Anal. Anw. 4, 305-329 (1985). MR 87a:76078

[32] M. Feistauer and A. Ženíšek, "Finite element solution of nonlinear elliptic problems," $N u$ mer. Math. 50, 451-475 (1987). MR 88f:65195

[33] G.J. Fix and G. Strang, An Analysis of the Finite Element Method, Prentice Hall, Englewood Cliffs, N.J. (1973). MR 56:1747

[34] F. I. Frankl and M. Keldysh, "Die äußere Neumannsche Aufgabe für nichtlineare elliptische Differentialgleichungen mit Anwendungen auf die Theorie der Flügel im kompressiblen Gas," Bull. Acad. Sci. URSS 12, 561-607 (1934).

[35] G. N. Gatica and G. C. Hsiao, "The coupling of boundary element and finite element methods for a nonlinear exterior boundary value problem," Numer. Math. 61, 171-214 (1992).

[36] R. Glowinski and O. Pironneau, "On the computation of transonic flows," in Funct. Anal. and Num. Anal., H. Fujita (ed.), Jap. Soc. Prom. Sci., Tokyo-Kyoto, 143-173 (1978).

[37] U. Göhner and G. Warnecke, "A shock indicator for adaptive transonic flow computations", Numer. Math. 66, 423-448 (1994). MR 95a:65199

[38] G. C. Hsiao and W. L. Wendland, "A finite element method for an integral equation of the first kind", J. Math. Anal. Appl. 58, 449-481 (1977). MR 57:1945

[39] C. Johnson and J. C. Nedelec, "On the coupling of boundary integral and finite element methods," Math. Comp. 35, 1063-1079 (1980). MR 82c:65072

[40] B. L. Keyfitz and G. Warnecke, "The existence of viscous profiles and admissibility for transonic shocks," Commun. Part. Diff. Eqns. 16, 1197-1221 (1991). MR 92i:76063

[41] N. Kroll and R. K. Jain, Solution of Two-Dimensional Euler Equation Experience with a Finite Volume Code, DFVLR-Forschungsbericht 87-41, DFVLR Institut für Entwurfsaerodynamik, Braunschweig 1987.

[42] L. D. Landau and E. M. Lifschitz, Lehrbuch der theoretischen Physik VI - Hydrodynamik, Akademie Verlag, Berlin (1981). MR 93b:00001c

[43] M. N. LeRoux, Résolution numérique du problème du potential dans le plan par une méthode variationelle d'éléments finis, Doctoral Thesis, University Rennes, 1974.

[44] J. Mandel and J. Nečas, "Convergence of finite elements for transonic potential flows", SIAM J. Numer. Anal. 24, 985-996 (1987). MR 89a:65170

[45] C. S. Morawetz, "Non-Existence of Transonic Flow Past a Profile I", Commun. Pure Apll. Math. 9, 45-68 (1956). MR 17:1149d

[46] C. S. Morawetz, "Non-Existence of Transonic Flow Past a Profile II", Commun. Pure Appl. Math. 10, 107-131 (1957). MR 19:490e

[47] C. S. Morawetz, "Non-Existence of Transonic Flow Past a Profile III", Commun. Pure Appl. Math. 17, 357-367 (1964). MR 32:1994

[48] C. S. Morawetz, "On a Weak Solution for a Transonic Flow Problem", Cummun. Pur Appl. Math. 38, 797-818 (1985). MR 87c:76087

[49] F. Murat, "L'injection du cône positiv de $H^{-1}$ dans $W^{-1, q}$ est compact pour tout $q<2$," J. Math. Pures Appl. 60, 309-322 (1981). MR 83b:46045

[50] J. Nečas, Compacite par Entropie et Ecoulements de Fluides, Lecture Notes Université de Charles et E.N-S., Masson, Paris (1989).

[51] M. Pogu and G. Tournemine, "Sur une classe de problèmes monotones posés dans des ouverts non bornés, méthode d'approche, algorithmes de résolution et application," Rev. Roumaine Math. Pures Appl. 31, 317-341 (1986). MR 88a:35051

[52] R. Rannacher and R. Scott, "Some optimal error estimates for piecewise linear finite element approximations". Math. Comp. 38, 437-445 (1982). MR 83e:65180 
[53] A. Rizzi and H. Viviant, Eds., Numerical Methods for the Computation of Inviscid Transonic Flows with Shock Wawes - Notes on Numerical Fluid Mechanics III, Vieweg, Braunschweig, 1986. MR 83j:76002

[54] J. Smoller, Shock Waves and Reaction-Diffusion Equations, Springer-Verlag, Berlin (1983).

[55] I.L. Sofronov and S.V. Tscyncov, "Application of the linear potential flow model to the artificial boundary conditions construction for the Euler equations." (Preprint No. 41 Nat. Center of Math. Simulation, Russian Acad. Sci. Moscow, 1991.) To appear.

[56] G. Warnecke, "Admissibility of solutions to the Riemann problems for systems of mixed type - transonic small disturbance theory," In: Nonlinear Evolution Equations that Change Type, B. L. Keyfitz and M. Shearer (eds.), IMA-Series Volume 27, Springer-Verlag, New York, 258-284 (1990). MR 91f:35187

[57] J. Zierep, Theoretische Gasdynamik, Braun, Karlsruhe (1976).

Braunag F-1 TW4, Frankfurter Str 145, D-61476 Kronberg, Germany

Fakultät für Mathematik, Otto-Von-Guericke-Universität Magdeburg, PF 4120, D39016 Magdeburg, Germany

E-mail address: gerald.warnecke@mathematik.uni-magdeburg.de

Mathematisches Institut A, Universität Stuttgart, Pfaffenwaldring 57, D-70569 Stuttgart, Germany

E-mail address: wendland@mathematik.uni-stuttgart.de 